\title{
Exploration du rôle potentiel des pharmacies privées dans l'offre de services de planification familiale au Sénégal: Recherche qualitative
}

\author{
Fatou Mbow \\ Population Council \\ Nafissatou Diop \\ Population Council \\ Sara Chace Dwyer \\ Population Council \\ Fatou Ndiaye \\ Bocar Mamadou Daff
}

Follow this and additional works at: https://knowledgecommons.popcouncil.org/departments_sbsr-rh How does access to this work benefit you? Let us know!

\section{Recommended Citation}

Mbow, Fatou, Nafissatou Diop, Sara Chace Dwyer, Fatou Ndiaye, and Bocar Mamadou Daff. 2018.

"Exploration du rôle potentiel des pharmacies privées dans l'offre de services de planification familiale au Sénégal: Recherche qualitative." Dakar: Population Council, The Evidence Project. 
Exploration du rôle potentiel des pharmacies privées dans l'offre de services de planification familiale au Sénégal

Fatou Bintou Mbow

Nafissatou Diop

Sara Chace Dwyer

Fatou Ndiaye

Bocar Mamadou Daff

RECHERCHE QUALITATIVE

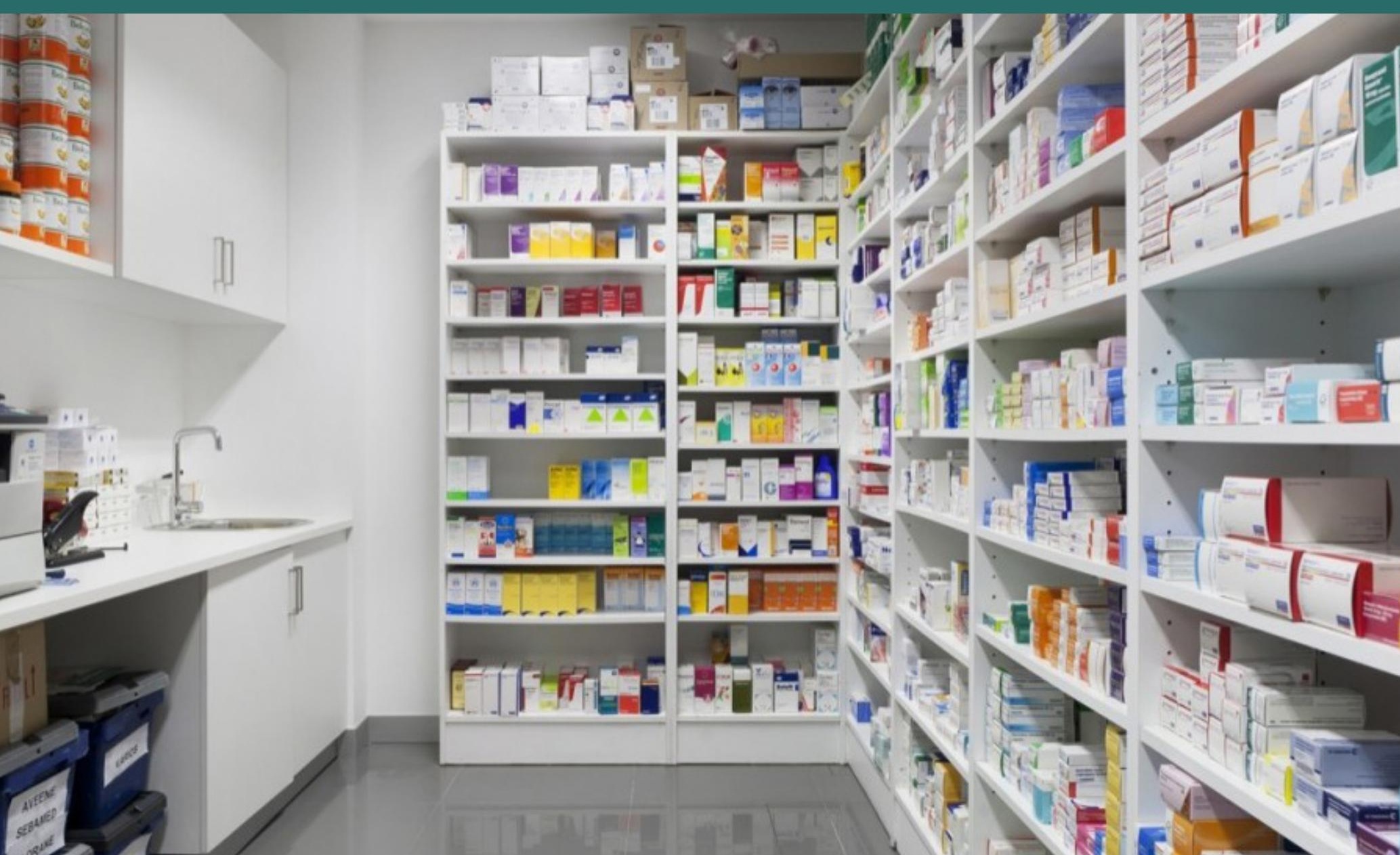





\section{Exploration du rôle potentiel des pharmacies privées dans l'offre de services de planification familiale au Sénégal}

\section{RECHERCHE QUALITATIVE}

Fatou Bintou Mbow, Population Council

Nafissatou Diop, Population Council

Sara Chace Dwyer, Population Council

Fatou Ndiaye, USAID Senegal

Bocar Mamadou Daff, Ministère de la Santé et de l'Action Sociale du Sénégal 
USAID Le Projet Evidence est rendu possible grâce au généreux support du Peuple Américain à travers l'Agence des Etats Unis pour le Développement International (USAID) sous les termes de l'accord de coopération no. AIDOAA-A-13-00087. Le contenu de ce document est la seule responsabilité du Projet Evidence et ne reflète pas nécessairement les points de vue de I'USAID ou du Gouvernement des Etats Unis. Evidence $\begin{aligned} & \text { Le Projet Evidence utilise la science de la mise } \\ & \text { en œuvre -la production, traduction, et utilisation }\end{aligned}$ stratégique d'évidences - pour renforcer et mettre à l'échelle des programmes de planification familiale et de santé de la reproduction afin de réduire les grossesses non désirées partout dans le monde. Le Projet Evidence est dirigé par le Population Council en partenariat avec Population Reference Bureau,

\footnotetext{
II wiliam + Flora La Fondation William et Flora Hewlett est une fondation de Hewlett bienfaisance non partisane et privée qui avance des idées Foundation et soutient les institutions pour promouvoir un monde meilleur.
}

\section{The Evidence Project}

Population Council

Sacré Cœur Pyrotechnie

Appartement 2ème Etage à Droite

Dakar

Sénégal

21027 Dakar Ponty

tél: +221.33.859.5300

evidenceproject@popcouncil.org

Publié en Mai 2018.

Citation recommandée: Mbow, Fatou Bintou, Nafissatou Diop, Sara Chace Dwyer, Fatou Ndiaye, et Bocar Mamadou Daff. 2018. "Exploration du rôle potentiel des pharmacies privées dans l'offre de services de planification familiale au Sénégal", Revue documentaire sur les évidences non juridiques. Dakar, Sénégal: Population Council, Le Projet Evidence.

(c) 2018 Le Population Council, Inc. 


\section{Table des Matières}

Remerciements................................................................................... vi

Acronymes et abréviations .................................................................. vii

Résumé exécutif .............................................................................. 1

Executive summary (English) ............................................................. 4

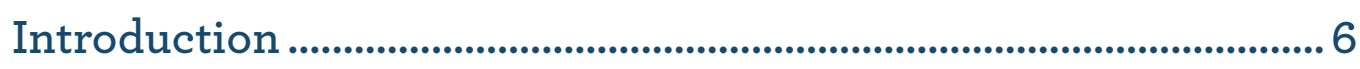

Contexte et justification ......................................................................... 7

Objectifs ......................................................................................................... 9

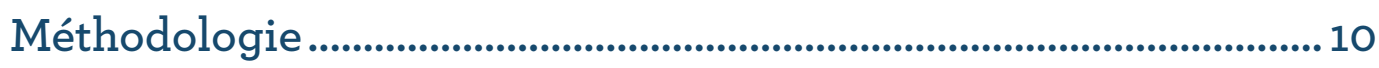

Résultats ........................................................................................................... 15

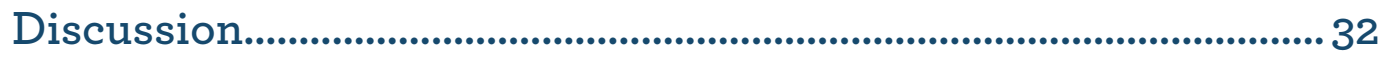

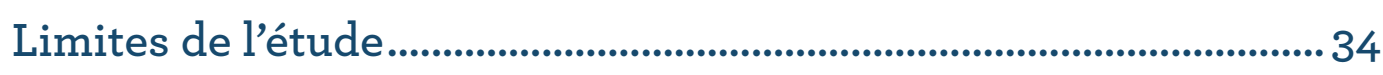

Recommandations ........................................................................................ 35

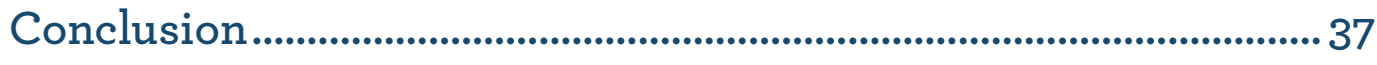

Références bibliographiques ......................................................... 39 


\section{Remerciements}

Nous remercions toutes les institutions, organisations et personnes qui ont contribué à la réalisation de cette étude en mettant à notre disposition la documentation utile ou en nous orientant vers d'autres parties prenantes susceptibles d'en détenir.

Nous voulons aussi témoigner notre profonde gratitude à toutes les parties prenantes intervenant dans le domaine de la planification familiale et aux pharmacies privées pour leur précieuse collaboration à travers leur facilitation pour la mise en œuvre de l'étude et les réponses apportées à nos questions. Nous saluons le professionnalisme des agents de collecte des données qui a beaucoup contribué à la réussite de cette étude.

Cette recherche a été réalisée grâce au soutien financier de I'USAID à travers le Projet Evidence, et l'appui de la Fondation William et Flora Hewlett à travers un financement au Population Council. 


\title{
Acronymes et abréviations
}

\author{
ADEMAS Agence pour le Développement du Marketing Social \\ ASC Agent de santé communautaire \\ BNS Besoins non satisfaits \\ CNOPS Conseil National de l'Ordre des Pharmaciens du Sénégal \\ CU Contraception d'urgence \\ DIU Dispositif Intra Utérin \\ DSRSE Direction de la Santé de la Reproduction et de la Survie de l'Enfant \\ EA Entretiens individuels approfondis \\ EDS-C Enquête Démographique et de Santé Continu \\ MLE Monitoring, Learning and Evaluation \\ ONG Organisation non gouvernementale \\ PANPF Plan d'action national de Planification Familiale \\ PF Planification familiale \\ PNA Pharmacie nationale d'approvisionnement \\ PPP Partenariat public-privé \\ PTF Partenaire technique et financier \\ SR Santé de la reproduction \\ SR/PF Santé de la reproduction / planification familiale \\ TPCm Taux de prévalence contraceptive moderne \\ USAID Agence des Etats-Unis pour le Développement International
}





\section{Résumé exécutif}

En 2012, la Direction de la Santé de la Reproduction et de la Survie de l'Enfant (DSRSE) du Ministère de la Santé et de l'Action Sociale (MSAS) du Sénégal a adopté un ambitieux Plan d'Action National de Planification Familiale (PANPF) pour la période 2012-2015. Ce plan visait un taux de prévalence contraceptive moderne (TPCm) de 12\% en 2012 à $27 \%$ en 2015 et $45 \%$ pour 2020. Entre 2012 et 2014, le TPCm a fait des bonds annuels de quatre (4) points de pourcentage, pour atteindre 20\% (EDS-C 2015). Le rythme de progression a toutefois diminué en 2015 avec un TPCm de 21\% (EDS-C 2016). Malgré tout, les besoins non satisfaits (BNS) en planification familiale (PF) restent encore importants. Ils étaient estimés à 25\% en 2015 (EDS-C 2016) parmi les femmes en union. Pourtant sur la base de l'Approche 3D, les pays du Partenariat se sont engagés à démocratiser, à démédicaliser et à décentraliser certains services de PF. Cela a commencé par l'introduction de politiques de délégation des tâches en matière de santé génésique en général et de la PF en particulier.

Le PANPF 2012-2015 s'appuie sur une approche multisectorielle qui fait du niveau communautaire et du secteur privé, des partenaires clés du secteur public dans sa mise en œuvre. Cependant, le secteur privé à but lucratif, en particulier les pharmacies privées, ne participe pas activement à l'offre de services de PF du fait de contraintes juridiques et non-juridiques limitant leur rôle actuel au conseil et à la vente de produits contraceptifs. Ainsi, en 2015, une étude exploratoire sur le rôle potentiel des pharmacies privées dans l'offre de services de PF au Sénégal a été menée par le Population Council, en collaboration avec la DSRSE, le Bureau santé de l'Agence des États-Unis pour le Développement International (USAID) au Sénégal, l'Agence pour le Développement du Marketing Social (ADEMAS), I'Unité de Coordination du Partenariat de Ouagadougou et l'Institut de Population, Développement et Santé de la Reproduction de l'Université Cheikh Anta Diop de Dakar. Les évidences de cette étude pourront soutenir les efforts de plaidoyer en faveur d'une plus grande implication des pharmacies privées dans l'offre de services de PF dans le cadre de l'approche multisectorielle adoptée par le gouvernement du Sénégal afin de mieux répondre aux besoins de PF et de santé sexuelle et reproductive des personnes et des couples au Sénégal. La composante qualitative de cette étude qui fait l'objet du présent rapport a pour objectifs spécifiques d'explorer des options et solutions offertes aux intervenants clés pour une possible implication des pharmacies privées, et de contribuer à la production de données probantes pour le plaidoyer.

\section{Méthodologie}

Cinquante-sept entretiens individuels approfondis (EA) ont été menés auprès d'acteurs clés dont 27 parties prenantes de la PF (parlementaires, représentants du gouvernement, partenaires au développement, représentants d'organisations non gouvernementales (ONG), membres de la société civile, représentants d'associations/réseaux professionnels et distributeurs pharmaceutiques) dans la région de Dakar et 30 pharmaciens dans les villes de Dakar, Pikine et Guédiawaye. Ces EA ont été complétés par la collecte de données sur le conseil/counseling et les ventes de produits de PF dans les pharmacies privées lorsqu'elles étaient disponibles. L'analyse de contenu a été utilisée pour l'interprétation des données. 


\section{Résultats}

L'analyse des données a montré que les pharmaciens ont une expérience essentiellement dans le conseil et la vente de produits contraceptifs. Pour certaines méthodes telles que les contraceptifs oraux (CO) et les injectables, ainsi que les méthodes de longue durée, les femmes sont le plus souvent orientées vers les structures de santé publique, ou quelques fois vers des cabinets privés. Toutefois, lorsque la femme est une personne connue, dans certains cas (réapprovisionnement ou situation d'urgence), les pharmaciens dérogent à la règle et offrent directement le service. Certains de ceux qui ont participé à des expériences pilotes ou interventions initiées par des ONG peuvent faire du counseling et la référence de clientes.

Mais, les pharmacies privées sont limitées dans leurs prestations par des textes réglementant le secteur privé, certaines dispositions du code de déontologie régissant la profession et la politique pharmaceutique et règlementant la vente de médicaments tels que certains produits contraceptifs. Concernant les contraintes non juridiques, il a été relevé ce qui suit : les pharmaciens manquent ou n'ont pas suffisamment de formation sur les technologies contraceptives ; la configuration des locaux ne permet pas toujours de garantir la confidentialité devant accompagner l'offre de services de PF ; les pharmacies privées ne disposent pas de toute la gamme de méthodes contraceptives offertes par le Programme PF et font quelque fois face à des ruptures de stock de certaines marques de $\mathrm{CO}$.

En même temps, la majorité des personnes interrogées, y compris les pharmaciens eux-mêmes, sont favorables à l'idée d'impliquer les pharmacies privées dans l'offre de services de PF. Plusieurs arguments ont été invoqués : cela pourrait contribuer à améliorer les performances des programmes de santé de la reproduction / planification familiale (SR/ $\mathrm{PF}$ ) et certaines pharmacies administrent déjà des méthodes de PF de manière non-officielle ; certaines méthodes de PF sont offertes par des agents moins qualifiés que les pharmaciens ; et cela pourrait renforcer le partenariat public-privé (PPP).

La majorité des répondants pense aussi que, hormis le pharmacien ou son assistant, certains vendeurs/agents de comptoir, qu'ils assimilent à du personnel communautaire, s'ils sont bien formés, peuvent également offrir des services de PF. Non seulement ils constituent souvent la majorité du personnel des pharmacies privées, mais les pharmaciens étant généralement absents, ce sont les vendeurs qui sont le plus souvent en contact avec les populations. En plus du rôle de conseiller qu'ils jouent déjà, les pharmaciens privés pourraient faire le counseling et même être impliqués dans l'offre initiale de méthodes contraceptives, mais seulement pour celles de courte durée telles que les pilules et les injectables.

Par ailleurs, l'implication des pharmacies privées dans l'offre de services de PF est considérée comme une stratégie à mettre en œuvre du fait des nombreuses opportunités offertes : les pharmacies constituent souvent le premier recours aux soins des populations ; elles disposent déjà d'une expérience dans la vente de produits contraceptifs ; le nombre important des pharmacies privées ; la disponibilité du personnel par rapport aux horaires d'ouverture ; l'intérêt manifesté par les pharmaciens et autres acteurs clés de la PF de voir les pharmacies privées jouer un plus grand rôle dans ce domaine. 
Toutefois, les défis à relever pour l'offre de services de PF par les pharmacies privées sont de divers ordres, notamment : la révision des lois et règlements ; la formation ; la disponibilité des produits/approvisionnement ; le réaménagement des pharmacies privées ; la collecte des données et leur transmission ; le suivi/supervision des pharmacies.

\section{Conclusion}

La non-participation des pharmacies privées à l'offre de services de PF résulte d'un environnement juridique et règlementaire limitant et de contraintes non juridiques. L'implication des pharmacies privées dans l'offre de services de PF doit devenir une réalité, conformément aux engagements nationaux et internationaux du Sénégal, à l'Approche 3D et aux autres recommandations de démédicalisation, d'autant que l'Organisation Mondiale de la Santé encourage une plus grande délégation des tâches au personnel non médical. Afin de poursuivre les efforts visant à promouvoir cette pratique prometteuse à haut impact, un plaidoyer fort et urgent est nécessaire pour optimiser le rôle des pharmaciens dans l'amélioration de l'accès aux services de PF. L'idée est acceptable par les parties prenantes de la PF et les pharmaciens eux-mêmes, à condition que les obstacles soient levés. La prochaine étape clé serait une étude pilote pour en explorer la faisabilité. 


\section{Executive summary (English)}

In 2012, the Directorate of Reproductive Health and Child Survival (DRSE) of the Ministry of Health and Social Action of Senegal adopted the ambitious National Family Planning Action Plan for 2012-2015. The plan had a target of reaching a modern contraceptive prevalence rate of $12 \%$ by $2012,27 \%$ by 2015 and $45 \%$ by 2020 . Between 2012 and 2014 , the modern contraceptive prevalence rate increased four percentage points annually, reaching $20 \%$ in 2014 (DHS-C 2015). It increased only to 21\% in 2015 (DHS-C 2016) and the unmet need for family planning remained high (25\%) among women in union in 2015 (EDS-C 2016). Based on the 3D Approach, Senegal and other Ouagadougou Partnership countries have committed to democratizing, de-medicalizing, and decentralizing certain family planning services. This has led to the introduction of task-shifting policies in reproductive health generally, and for family planning specifically.

The 2012-2015 National Family Planning Action Plan used a multi-sectoral approach that involved the private sector and community level as key partners with the public sector in implementing these plans. However, private pharmacies have not been actively involved in the provision of family planning services due to legal and non-legal barriers that limit their role to selling contraceptives with a prescription and providing method-specific advice. In 2015, the Population Council, in collaboration with the DSRSE, the U.S. Agency for International Development (USAID) Senegal Health Office, the Agency for the Development of Social Marketing, the Ouagadougou Partnership Coordination Unit, and the Institute of Population, Development and Reproductive Health, Cheikh Anta Diop University of Dakar, conducted an exploratory study on the potential role of private pharmacies in the provision of family planning services in Senegal. The study was funded by USAID, through the Evidence Project, and the William and Flora Hewlett Foundation. As part of this study, qualitative data were collected from key stakeholders and private pharmacists to explore the current situation, including barriers and solutions, and to advocate for greater involvement of private pharmacies.

\section{Methodology}

Fifty-seven in-depth interviews were conducted, 27 with family planning (FP) stakeholders in the Dakar region (Parliamentarians, government representatives, development partners, non-governmental organization representatives, civil society representatives, association/network representatives, and pharmaceutical distributors) and 30 with pharmacists from Dakar, Pikine and Guédiawaye cities. In addition to the in-depth interviews, data were collected on the sale of family planning products and instructions provided to clients in private pharmacies, where available.

\section{Results}

Private pharmacists had experience selling contraceptive methods and providing method-specific advice. If the pharmacist knew a client, she was provided with a contraceptive method without prescription (typically for a refill or in an emergency). Private pharmacies are limited in their ability to provide family planning services by laws regulating the private sector, the pharmacist's professional code of conduct, and pharmaceutical policies regulating drug sales (which include contraceptives). Concerning the non-legal constraints, the results are 
that pharmacists had insufficient training in contraceptive technology, pharmacies lacked adequate private space for family planning counseling, and some private pharmacies did not have the full range of contraceptive methods available and often faced stock-outs.

Most respondents, including the pharmacists themselves, support the idea of involving private pharmacies more in the provision of family planning services. Common themes include : private pharmacies' involvement could contribute to improve the performance of reproductive health and family planning programs; some pharmacies are already providing FP services, although illegally; certain family planning methods are currently offered by less educated providers than pharmacists; and private pharmcies' involvement could strengthen broader public-private partnership efforts.

Most respondents believe that pharmacy counter agents (who are similar to community health workers), if trained, could also offer family planning services. Counter agents are the pharmacy staff most often available, as pharmacists are generally not in their pharmacy. Therefore, counter agents are most often in contact with customers. In addition to the guidance they already provide on drug indications, private pharmacists could counsel clients on family planning and be involved in the initial provision of short-acting contraceptives such as pills and injectables.

There are many reasons to involve private pharmacies in the provision of FP services. Private pharmacies are often the first point of care for the population and there are many pharmacies present, especially in the Dakar region. They also already have experience selling contraceptives and have staff available for extended hours. Finally, both private pharmacists and key family planning actors are interested in exploring how private pharmacies can play a more important role in this area.

While there are opportunities, there are also challenges. Some laws and regulations would need to be revised. Pharmacy staff would require training. Pharmacies would need adequate contraceptive methods available and private spaces for family planning counseling, and mechanisms for collecting family planning data and sharing that data with district health offices.

\section{Conclusion}

The non-participation of private pharmacies in the provision of family planning services is a result of legal and non-legal constraints. Including private pharmacies in the provision of family planning service provision is in accordance with Senegal's national and international commitments, its 3D approach (demedicalization, democratiziation, and decentralization) and with other global task-shifting initiatives, including recent recommendations from the World Health Organization. To promote this high-impact promising practice, strong and urgent advocacy is needed to increase the role of pharmacists in improving clients' access to FP services. The idea is acceptable to FP stakeholders and pharmacists themselves, provided the barriers are removed. The next key step would be a study to explore the feasibility of private pharmacy provision of family planning services. 


\section{Introduction}

Au Sénégal, l'indice synthétique de fécondité (ISF) s'est stabilisé autour de 5 enfants par femme entre l'enquête démographique et de santé à indicateurs multiples de 2005 et l'enquête démographique de santé continue (EDS-C) de 2014. Par contre, les taux de mortalité maternelle et infanto-juvénile ont considérablement diminué, passant respectivement de 401 à 392/100 000 naissances vivantes et de 121 à 54/1 000 pendant la même période.

Le taux de prévalence contraceptive moderne (TPCm), après avoir augmenté timidement jusqu'en 2010, a fait des bonds annuels de quatre (4) points de pourcentage à partir de 2012, pour atteindre 16\% entre 2012 et 2013 et 20\% en 2014 (EDS-C 2014). Le rythme de progression a toutefois diminué en 2015 avec un TPCm rapporté de 21\% (EDS-C 2015). Malgré tout, les besoins non satisfaits (BNS en PF restent importants. Par exemple, parmi les femmes en union, ils étaient estimés à 25\% en 2015 (EDS-C 2015).

Au Sénégal, notamment en milieu urbain, le nombre élevé, la proximité géographique et les horaires étendus des pharmacies ${ }^{1}$ en font souvent les premiers points de contact des populations avec le système de santé. Cependant leur potentiel d'offre de services de PF n'est pas suffisamment exploité. Selon l'EDS-C 2015, pour l'obtention des méthodes modernes de contraception, $84 \%$ des femmes sénégalaises s'adressaient au secteur public, mais un peu plus d'une femme sur dix, soit 11\%, avaient recours au secteur privé et le reste (seulement 1\%) s'orientait vers d'autres sources. Dans ce secteur privé, les pharmacies, qui représentent $9 \%$, sont de loin les principaux fournisseurs. Elles constituent ainsi, en milieu urbain, la deuxième source d'approvisionnement après les établissements du secteur public.

1 Au Sénégal, la dénomination dépend du secteur. Dans le secteur privé, en milieu urbain, les pharmacies sont dénommées " officines de pharmacie ", plus communément appelées " officines ", ou " pharmacies privées ". En milieu rural, il y a les dépôts privés de médicaments. Dans le secteur public, l'appellation change suivant le niveau de la pyramide sanitaire : au niveau national, il y a la PNA ; chaque région (11 régions/14) dispose d'une " Pharmacie régionale d'approvisionnement "; les districts sanitaires disposent d'un " dépôt de district "; et au niveau des centres et postes de santé, on trouve un "dépôt " (de centre de santé, ou de poste). Les cases de santé sont desservies par le dépôt du poste de santé. Dans ce document "pharmacie " ou " pharmacie privée " renvoie au secteur privé. 


\section{Contexte et justification}

En 2012, la Direction de la Santé de la Reproduction et de la Survie de l'Enfant (DSRSE) du Ministère de la Santé et de l'Action Sociale (MSAS) a adopté un ambitieux Plan d'action national de Planification Familiale (PANPF) pour la période 2012-2015. Ce PANPF visait un accroissement du TPCm de $12 \%$ en 2012 à $27 \%$ en 2015, et 45\% en 2020. Cet objectif a été fixé conformément à l'engagement du pays d'atteindre les objectifs du millénaire pour le développement, en particulier les objectifs 4 et 5 . Le MSAS a renouvelé cet engagement à la déclaration FP2020 au Sommet de Londres en 2012. Le PANPF a adopté une approche multisectorielle faisant du secteur privé (à but lucratif et non lucratif) et du communautaire, des partenaires clés du secteur public dans sa mise en œuvre. De ce fait, sur les 350000 nouvelles utilisatrices de PF prévues, 100000 devraient être servies par le secteur privé et 125000 au niveau communautaire (PANF, 2012).

Depuis 2012, le Programme PF a fait des progrès considérables grâce en partie à l'implication active du niveau communautaire et des organisations du secteur privé à but non lucratif dans la promotion et l'offre de services de PF. Cependant l'objectif des $27 \%$ de TPCm n'a pas été atteint en 2015, l'ESD-C rapportant un taux de 21\%. Cette situation est essentiellement due au fait que le secteur privé à but lucratif, y compris les pharmacies privées, n'a pas été véritablement impliqué dans l'offre de services car la règlementation limite leur intervention en matière de PF à la vente des produits contraceptifs et à la fourniture de conseils ${ }^{2}$ aux utilisateurs potentiels. Les pharmacies privées sont donc exclues de l'offre effective des services de PF et ne peuvent qu'orienter les utilisateurs potentiels vers une structure publique de santé, un établissement du secteur privé à but non lucratif ou un médecin privé. Ceci est un obstacle à l'accès de certaines personnes et de certains couples à la PF. Pourtant, des expériences à travers le monde ont indiqué qu'au-delà d'être une source d'approvisionnement, les pharmacies privées pouvaient jouer un rôle important dans l'offre de services. Par ailleurs, au niveau global, les évidences soutiennent un plus grand rôle des pharmacies et dépôts des médicaments dans I'offre des services du PF (USAID, 2013). Par exemple, au Népal, l'EDS 2011 a montré que 12\% des femmes obtenaient leurs méthodes injectables dans les pharmacies, 52\% y achetaient des préservatifs et 32\% s'y approvisionnaient en pilules. De même, une étude de 2011 au Nigeria et en Ouganda, a montré que l'élargissement de la prestation de PF au secteur privé à but lucratif a permis de réduire les inégalités d'accès à la contraception moderne (Hotchkiss, 2011). En effet, l'EDS-2013 du Nigéria a montré que $38 \%$ des utilisatrices de la PF achetaient leurs produits auprès de vendeurs de médicaments brevetés ${ }^{3}$ qui constituent la première source de PF.

Dans le cadre du Projet Evidence financé par I'USAID, le Population Council a entrepris en collaboration avec la DSRSE et I'USAID Sénégal, une étude intitulée "Exploration du rôle potentiel des pharmacies privées dans l'offre de services de PF au Sénégal ". Les évidences de cette étude pourront soutenir les efforts de plaidoyer en faveur d'une plus grande

2 Le " conseil " peut être défini ici comme une information donnée par rapportà un mal bénin ou une préoccupation particulière. Par contre le " counseling " est un élément clé des services de PF. II s'agit d'un mécanisme qui se fait en plusieurs étapes et qui permet à la cliente d'obtenir de manière sûre et confidentielle toutes les informations relatives aux différentes méthodes de contraceptions disponibles afin de pouvoir faire un choix éclairé.

3 Au Nigéria, les vendeurs de médicaments brevetés sont des agents de la santé à la retraite, comme des assistants en pharmacie, des infirmières ou parfois des agents de santé communautaire qui vendent des médicaments après l'obtention d'une licence d'ouverture délivrée par le Conseil des pharmacies. 
implication des pharmacies privées dans l'offre de services de PF, dans le cadre de l'approche multisectorielle adoptée par le Gouvernement du Sénégal afin de mieux répondre aux besoins de PF et de santé sexuelle et reproductive des personnes et des couples au Sénégal. Elle intègre plusieurs composantes, à savoir une revue documentaire des aspects juridiques (défis et opportunités) pour l'offre de services de PF par les pharmacies privées, une revue documentaire des aspects non juridiques (y compris une analyse secondaire des données disponibles sur les pharmacies privées), une analyse secondaire des données issues d'études menées par l'Agence pour le développement du marketing social (ADEMAS), une organisation spécialisée en marketing social, et une recherche qualitative auprès des parties prenantes clés de la PF et des pharmaciens privés de la région de Dakar. Chaque composante a fait l'objet d'un rapport spécifique (les trois autres composantes ainsi que la synthèse de l'étude sont disponibles sur le site du Projet Evidence). Le présent rapport porte sur la composante "recherche qualitative". 


\section{Objectifs}

L'étude visait à fournir des évidences afin de soutenir le plaidoyer en faveur d'une plus grande implication des pharmacies privées dans l'offre de services de PF. La recherche qualitative a eu pour objectifs de :

- Explorer des options et solutions possibles avec des intervenants clés dans une possible implication des pharmacies privées; et

- Contribuer à fournir les évidences pour le plaidoyer. 


\section{Méthodologie}

Des entretiens individuels approfondis (EA) semi-structurés ont été menés dans la région de Dakar par une équipe d'agents de collecte formée à cet effet. Ces EA ont été complétés en juillet 2016 par la collecte de données sur le conseil/counseling et les ventes de produits de PF dans les pharmacies privées.

\section{Population cible}

Certains EA ont été menés avec 27 parties prenantes clés de la PF, y compris des décideurs politiques, des représentants du gouvernement, des partenaires au développement, des représentants d'ONG et de la société civile, des membres d'associations professionnelles, des distributeurs de produits pharmaceutiques, et autres. D'autres EA ont été menés avec 30 pharmaciens privés, y compris le pharmacien ou son assistant, le gérant de pharmacie, ou les vendeurs/agents de comptoirs.

\section{Sites}

La région de Dakar a été choisie, en collaboration avec la DSRSE, pour plusieurs raisons: elle est la capitale du pays et regroupe la plupart des institutions et organisations nationales et internationales intervenant dans le Programme PF. Elle abrite également plus de 50\% des pharmacies privées et $22 \%$ des habitants du pays. Les villes de Dakar, Pikine et Guédiawaye ont aussi été ciblées, en raison notamment de la grande densité de leurs populations.

\section{Procédures de collecte des données et de recrutement}

\section{Outils de collecte}

Trois (3) outils ont été élaborés en vue de : i) obtenir une meilleure compréhension des attitudes par rapport à l'implication des pharmacies privées dans l'offre de services de PF ; ii) aider à identifier les obstacles, les opportunités et les défis ; iii) étudier les solutions possibles. Ces outils sont les suivants :

- Le guide EA avec les parties prenantes de la PF.

- Le guide EA avec les pharmaciens privés.

- Le formulaire de collecte de données sur le conseil/counseling et les ventes de produits de PF.

Les 2 guides ont abordé les sujets suivants: 1) l'expérience de l'individu et son implication dans la PF ; 2) la perception de l'engagement des pharmacies privées dans l'offre de services de PF ; 3) la perception des opportunités et des défis ; 4) la perception de qui au sein de la pharmacie pourrait être autorisé à offrir les services ; 5) la perception de la formation requise pour pouvoir offrir les services de PF ; 6) les contraintes perçues à l'offre des services de PF dans une pharmacie privée ; et 7 ) les solutions pouvant rendre réelle leur implication dans l'offre de service de PF dans un avenir proche au Sénégal. 
Tous les outils ont fait l'objet de pré-tests avec les parties prenantes et les pharmaciens qui n'étaient pas inclus dans l'étude. Ensuite, les outils ont été finalisés en prenant en compte les remarques et suggestions pertinentes afin de mieux affiner les questions.

\section{Profil des agents de collecte}

La recherche qualitative a été conduite par deux (2) équipes (une pour chaque population cible). Chaque équipe était composée de trois (3) interviewers/intervieweuses ayant une expérience avérée en matière de recherche qualitative, et sélectionnés à l'issue d'une (1) formation spécifique regroupant cinq (5) candidats. Les agents devaient avoir une bonne maîtrise du français et de l'Ouolof, et une compétence en transcription d'interviews.

\section{Formation des agents de collecte}

Chaque équipe a reçu une formation théorique d'une demi-journée et une formation pratique étalée sur deux jours et demi. Cette formation avait essentiellement pour but de permettre aux agents de collecte d'avoir une compréhension commune des objectifs et de se familiariser avec les outils. Une présentation sur l'éthique en matière de recherche a permis de rappeler les principes fondamentaux qui gouvernent la recherche ainsi que les obligations du chercheur et les droits des participants. Une session a également été consacrée au rappel des principes et méthodes de recherche qualitative. La formation a aussi insisté sur les exigences liées à la collecte et à la gestion des données, avec un accent particulier sur l'anonymat et la confidentialité qui doivent être respectés durant tout le processus pour garantir une recherche de qualité. Aussi, des recommandations et précisions ont été faites sur la manière d'administrer les outils et la démarche à suivre pour être conforme au protocole.

Les outils devant être administrés au niveau des pharmacies privées ont été traduits oralement en Ouolof (langue nationale la plus utilisée au Sénégal) pour le cas où l'interviewé aurait le profil adéquat pour participer à la recherche, mais n'aurait pas un niveau de français suffisant pour un entretien productif dans cette langue.

La majeure partie du temps de formation a été consacrée aux exercices de simulation d'interviews et au prétest des outils. Ces activités étaient destinées, non seulement à tester les outils, mais aussi les compétences et aptitudes des participant(e)s pour les interviews.

\section{Recrutement des participants et déroulement de la collecte}

\section{Entretiens individuels avec des parties prenantes de la PF}

La collecte des données s'est déroulée du 25 mai au 20 juillet 2016. Au début du processus, une lettre d'information de la DSRSE accompagnée d'un courriel formel du Population Council ont été envoyés à chaque participant potentiel (pour l'essentiel membres du comité technique PF) pour expliquer l'étude et solliciter un rendez-vous pour un entretien dans l'endroit de son choix. Pour les intervenants qui ne répondaient pas à la demande de rendezvous envoyée par courriel, un suivi a été effectué par téléphone. La collecte a cessé lorsque 
tous les intervenants contactés ont effectué leur EA, ont refusé l'entretien, ou n'ont pas répondu au courrier ou aux appels téléphoniques pendant la période de collecte.

Au début de chaque entretien, un formulaire de consentement éclairé a été lu et signé de manière volontaire par le participant. Les entretiens ont duré en moyenne une heure et ont été enregistrés avec la permission des participants. Tous les entretiens ont été menés en face à face.

\section{Entretiens individuels avec des pharmaciens privés}

La collecte s'est déroulée du 14 juin au 11 juillet 2016. Comme pour les parties prenantes de la PF, des lettres d'information ont été envoyées par la DSRSE au Conseil National de l'Ordre des Pharmaciens du Sénégal (CNOPS) et à la Direction de la Pharmacie et du Médicament. La correspondance au CNOPS avait également pour objet de solliciter son appui dans la préparation de la collecte des données auprès des pharmacies privées.

Ensuite, une rencontre a été organisée entre la chargée de programmes du Population Council et le Président du CNOPS pour lui présenter l'étude, demander son aide à l'identification des pharmacies privées et solliciter une lettre d'introduction auprès des pharmacies retenues.

La sélection des pharmacies s'est effectuée comme suit : la liste des pharmacies privées a été obtenue auprès de l'ADEMAS et validée par le président du CNOPS. Puis, avec l'aide du responsable des achats d'un des plus gros distributeurs pharmaceutiques de la place, des pharmacies ont été sélectionnées pour chacune des villes de l'étude (Dakar, Pikine et Guédiawaye) en fonction des sorties/commandes des produits de PF et/ou de la population couverte. Compte tenu de la possibilité que des pharmaciens ne pourront ou ne voudront pas participer, plus de 10 pharmacies ont été présélectionnées pour chaque ville.

Les pharmacies sélectionnées ont ensuite été contactées par l'équipe de recherche, en s'appuyant sur la lettre d'introduction du président du CNOPS, afin d'expliquer l'étude, recueillir l'intérêt à participer, identifier la meilleure personne à interviewer et convenir d'un rendez-vous. Pour certaines pharmacies, les rendez-vous ont été obtenus dès les premiers appels téléphoniques, mais pour d'autres, il a fallu se déplacer avec la lettre d'introduction pour obtenir un rendez-vous.

Au début de l'entretien, les intervieweurs ont expliqué à nouveau les objectifs de l'étude et ont lu le consentement éclairé, qui inclut l'autorisation d'enregistrer l'entretien et de recueillir des statistiques sur le counseling et les ventes de produits de PF.

Les EA ont été effectués uniquement avec les participants qui ont volontairement signé le formulaire de consentement. Ils ont duré en moyenne 45 minutes et ont tous été menés en face à face.

À l'issue de l'entretien proprement dit, et lorsque les données étaient disponibles, les intervieweurs ont rempli le formulaire de collecte de données sur les statistiques en matière de counseling et de ventes de produits de PF. 


\section{Suivi de la collecte}

Un suivi rapproché de chaque équipe a été effectué par le Population Council durant toute la collecte. Après chaque entretien, un compte-rendu avec les agents de collecte a été effectué pour identifier les difficultés rencontrées.

\section{Difficultés rencontrées lors de la collecte}

La collecte s'est déroulée sans contraintes majeures. II convient, toutefois, de relever au niveau des interviewés, certaines difficultés, notamment:

- La non-disponibilité de certaines parties prenantes du fait de nombreuses activités planifiées durant la même période, une situation qui a souvent occasionné des reports de rendez-vous ;

- Le refus de participation de certaines parties prenantes, notamment du secteur privé de la santé (y compris les pharmaciens, malgré la lettre introductive du CNOPS), en dépit des rendez-vous pris, et des réponses apportées aux questions posées lors de la planification des interviews ;

- La mobilité des pharmaciens. Pour certaines pharmacies privées, l'accès n'a pas été aisé du fait des nombreux déplacements des pharmaciens. En plus des appels téléphoniques, plusieurs allers et retours ont été nécessaires pour l'obtention des rendez-vous, et certains ont eu du mal à les respecter. Cette mobilité s'est également ressentie pendant les entretiens. En effet, presque tous les entretiens se sont déroulés au sein des pharmacies. De ce fait, les interviews étaient souvent entrecoupées afin de permettre à l'interviewé(e) de s'occuper des clients. Par ailleurs, l'accès aux informations sur les données relatives au counseling et aux ventes, pour les pharmacies qui en avaient, a parfois été difficile en raison de l'absence du pharmacien titulaire.

- L'affluence des clients à cause de laquelle certains rendez-vous ont été pris à des heures incongrues, notamment très tôt le matin.

- Le manque d'exhaustivité des données sur le conseil/counseling et les ventes, et parfois les lenteurs pour les obtenir. Dans certains cas, cet exercice a pris beaucoup de temps, et a parfois même, dû se poursuivre au téléphone pour la collecte de certaines données.

\section{Gestion des donnés}

Les EA ont été enregistrés puis transcrits sur Microsoft Word. Pour préserver l'anonymat des participants et la confidentialité de toute information qui permettrait l'identification des répondants et leur localisation, un système de cryptage a été établi. Par ailleurs, les transcriptions et les fichiers audio des EA ont été conservés dans un endroit sécurisé conformément aux critères de protection des données du Population Council. L'accès y a été réservé à la seule équipe de recherche habilitée. 


\section{Analyse des données}

L'analyse de contenu a été utilisée pour l'interprétation des données. Le contenu des discours des interviewés a été étudié pour faire ressortir les thèmes les plus souvent abordés, les mots clés, les prises de position et les arguments invoqués pour les justifier. Cette analyse a combiné une démarche descriptive et inductive pour d'une part présenter un état des lieux, et d'autre part déterminer les thèmes et les modèles qui ont ensuite permis de construire des typologies. Cela a permis de répondre de manière plus fine aux besoins de l'analyse.

\section{Aspects éthiques}

Le protocole de recherche, y compris le draft des outils de collecte, a reçu l'approbation éthique du Comité d'Ethique du Population Council et du Comité National d'Ethique pour la Recherche en Santé du MSAS, ainsi qu'une autorisation administrative de la Direction de la Planification, de la Recherche et des Statistiques du MSAS.

Le formulaire de consentement éclairé a été présenté à chaque participant potentiel. Ceux qui savaient lire, l'ont lu eux-mêmes, à leur demande. Pour tous les autres, le formulaire a été lu par l'intervieweur/intervieweuse. Les informations et exigences liées à la participation à l'étude ont été présentées. Le consentement libre et éclairé a ensuite été demandé. Seuls les participants qui ont exprimé leur accord et signé le formulaire de consentement ont été interviewés.

Plusieurs mesures ont été prises afin de garantir la confidentialité des EA : ils ont été réalisés par des intervieweurs expérimentés, dans des lieux les plus privés possibles ; le lieu précis n'est noté dans aucun document ; les informations électroniques ont été enregistrées sur des ordinateurs protégés par des mots de passe ; les notes écrites et les enregistrements audio ont été gardés dans des placards verrouillés au bureau de Population Council. Une fois que la transcription sera achevée et vérifiée et que le rapport d'étude sera publié, les notes écrites sur papier et les enregistrements audio des EA seront détruits.

Pour s'assurer de l'anonymat des répondants et des informations collectées, aucune identification n'a été enregistrée sur les notes d'entretien, les enregistrements ou les transcriptions électroniques. Les données ont été gardées séparément des identifiants. 


\section{Résultats}

\section{Bilan de la collecte des données}

Dans le cadre de cette étude, un total de 57 entretiens approfondis a été réalisé, dont 27 auprès des parties prenantes de la PF et 30 auprès des pharmaciens privés. Le tableau ci-dessous dresse le bilan détaillé.

\section{TABLEAU 1}

RÉCAPITULATIF DES PERSONNES INTERROGÉES

\begin{tabular}{|c|c|c|c|c|c|c|}
\hline \multirow{2}{*}{\multicolumn{2}{|c|}{ Population Cible }} & \multicolumn{3}{|c|}{ Site } & \multirow{2}{*}{ Total } & \multirow{2}{*}{ Total } \\
\hline & & Dakar & Guédiawaye & Pikine & & \\
\hline \multirow{7}{*}{ Parties prenantes de la PF } & Parlementaires & 2 & & & 2 & \multirow{7}{*}{27} \\
\hline & $\begin{array}{l}\text { Représentants du } \\
\text { gouvernement }\end{array}$ & 6 & & & 6 & \\
\hline & $\begin{array}{l}\text { Partenaires au } \\
\text { développement }\end{array}$ & 2 & & & 2 & \\
\hline & Représentants ONG & 7 & & & 7 & \\
\hline & $\begin{array}{l}\text { Membres de la société } \\
\text { civile }\end{array}$ & 3 & & & 3 & \\
\hline & $\begin{array}{l}\text { Membres d'association } \\
\text { professionnelle/réseaux }\end{array}$ & 4 & & & 4 & \\
\hline & $\begin{array}{l}\text { Distributeurs } \\
\text { pharmaceutiques }\end{array}$ & 3 & & & 3 & \\
\hline \multirow{2}{*}{$\begin{array}{c}\text { Personnel des pharmacies } \\
\text { privées }\end{array}$} & Pharmacien(ne)s & 9 & 9 & 10 & 28 & \multirow{2}{*}{30} \\
\hline & Pharmaciens assistants & 1 & 1 & 0 & 2 & \\
\hline Total & & & & & 57 & 57 \\
\hline
\end{tabular}

Malgré la diversité des profils, toutes les parties prenantes de la PF avaient plus de cinq (5) ans d'expérience dans le domaine. Cette expérience a été acquise en tant que gestionnaires de programmes (pour la plupart), décideurs politiques, médecins, fournisseurs de produits, bailleurs de fonds ou agents de plaidoyer. Au niveau des pharmacies, les rendez-vous ont été obtenus auprès des pharmaciens et pharmaciens assistants qui tenaient eux-mêmes à participer aux entretiens. Ils avaient tous en moyenne huit (8) années d'expérience.

\section{Expérience des pharmaciens dans le domaine de la PF}

En matière de PF, les pharmaciens ont une expérience essentiellement dans la délivrance ou la dispensation ${ }^{4}$ de produits contraceptifs. Selon eux, les méthodes de PF les plus

4 Dans le domaine pharmaceutique, c'est le terme " délivrance " qui est consacré, plutôt que celui de " vente ". Et " délivrance " est différente de " dispensation ", qui signifie délivrance accompagnée de conseils. Par exemple un agent de comptoir fait de la délivrance, mais il appartient au pharmacien de faire de la dispensation, pour les produits vendus sur ordonnance. 
dispensées dans les pharmacies privées sont les préservatifs, les contraceptifs oraux (surtout la contraception d'urgence [CU]) qui est désormais en vente libre, les injectables, et les spermicides. Lorsque les femmes souhaitent utiliser d'autres méthodes, elles sont le plus souvent référées vers les structures de santé publique, ou quelques fois vers des cabinets privés. Toutefois, plusieurs pharmaciens ont déclaré déroger à cette règle, dans certains cas, en offrant directement le service. C'est le cas par exemple lorsqu'il s'agit de réapprovisionnement, ou lorsque la femme est une personne connue du pharmacien, en témoigne les propos suivants :

"Nous essayons d'agir au cas par cas. Nous avons des femmes qui habitent le quartier, que nous connaissons bien, qui viennent pour renouveler. Et nous savons que c'est extrêmement difficile à chaque fin du mois d'aller voir la sage-femme ou le/la gynécologue pour renouveler son ordonnance. Mais si c'est une personne que nous ne connaissons pas, ou si nous pensons que la personne est mineure, là quand même nous refusons " [Pharmacien, Dakar].

"Pour les renouvellements nous leur donnons les produits qu'ils demandent. Nous connaissons les personnes qui généralement ont l'habitude d'acheter les produits chez nous, donc nous leur donnons sans ordonnance " [Pharmacien, Dakar].

Les pharmaciens ont indiqué que les méthodes disponibles dans les pharmacies privées étaient fonction de la demande, et de ce que les grossistes-répartiteurs distribuaient. Ainsi, ils ont déclaré que si les implants ou le Dispositif Intra-Utérin (DIU) n'étaient quasiment vendus dans aucune pharmacie privée ${ }^{5}$, c'est parce qu'ils ne recevaient pas d'ordonnances pour ces méthodes, qui sont par ailleurs considérées par certains comme des produits réservés au milieu hospitalier.

En outre, la plupart des parties prenantes de la PF interrogées notent une certaine évolution dans l'expérience des pharmacies privées en matière de PF, du fait des nombreuses rencontres techniques auxquelles leurs représentants sont conviés, ou encore de certaines interventions auxquelles elles sont associées. Pourtant l'analyse des données montre qu'en dehors des pharmaciens ayant participé récemment à des expériences pilotes ou interventions initiées par des $\mathrm{ONG}^{6}$ leur permettant de faire du counseling et la référence de clientes, la majorité d'entre eux ne se sentait pas impliquée dans l'offre de services de PF du fait de la limitation de leurs prestations dans ce domaine, puisque l'offre de service est restée quasiment au même niveau (délivrance, conseils et référence). Les propos de ce représentant d'ONG qui rapporte les propos de certains pharmaciens, en sont une parfaite illustration : "nous, on ne nous associe pas par rapport à cet effort de santé publique " disent-ils.

5 L'étude MAP 2015 de l'ADEMAS a montré que les implants étaient pratiquement absents des pharmacies privées visitées et que seuls $2 \%$ d'entre elles distribuaient le DIU.

6 Par exemple, l'expérience des pharmacies pilotes PF de Marie Stopes International menée dans le cadre du projet Initiative Sénégalaise de Santé Urbaine de Intrahealth, ou encore de certaines interventions de l'ADEMAS. 


\section{Obstacles à l'offre de services de PF par les pharmacies privées}

Plusieurs contraintes à l'offre de services de PF par les pharmacies privées ont été identifiées. Ils sont de divers ordres.

\section{Les contraintes juridiques}

Les informations recueillies montrent que les pharmacies privées sont d'abord régies par des textes réglementant le secteur privé et leur interdisant, tout comme aux autres établissements de ce secteur, de faire à la fois des prestations en matière de counseling, de prescription et de vente de produits contraceptifs. Certains de ces textes datent de 1954 et sont jugés désuets, notamment par les pharmaciens eux-mêmes. Ensuite, les pharmaciens privés sont astreints à un code de déontologie les empêchant d'effectuer certains actes médicaux, tels que les injections. Enfin, dans le cadre de la politique pharmaceutique, la vente des médicaments est très règlementée. Pour certains produits, la dispensation ne doit être faite que sur présentation d'une ordonnance. II s'agit notamment des produits des listes I et II $^{7}$ de la nomenclature internationale de classification des médicaments, dont font partie les $\mathrm{CO}$ et les injectables. Ainsi, pour la prescription d'un produit contraceptif inscrit sur une des listes, les femmes doivent préalablement consulter un médecin, un infirmier ou une sage-femme exerçant dans une structure sanitaire (publique ou privée). Elles peuvent ensuite amener l'ordonnance à la pharmacie privée pour délivrance. Par ailleurs, la Loi sur la SR de 2005 relative, entre autres, aux droits spécifiques des individus en matière de SR et PF traite pourtant en son Article $8^{8}$ du personnel pouvant fournir des services de SR. Mais cet article fait référence au respect de son code de déontologie par chaque catégorie professionnelle. C'est dire que les pharmaciens ont l'obligation de s'en tenir aux règles édictées par la réglementation pharmaceutique et également leur code de déontologie, ce qui fait dire à un pharmacien de Pikine que : "Un pharmacien qui respecte le code de déontologie, qui suit ce que les textes exigent, est toujours limité dans ces prestations".

La majorité des pharmaciens interrogés pensent que, pour être en phase avec les objectifs de PF à atteindre, il faut nécessairement revoir la législation. De plus, les textes créent des incompréhensions, voire des frustrations, dans la mesure où les pharmaciens privés sont interdits de pratiquer des choses pour lesquelles ils ont été formés dans leur cursus universitaire, notamment les injections. De même concernant les produits, la CU qui est beaucoup plus dosée que les autres contraceptifs oraux est délivrée sans ordonnance comme illustrée par les propos suivants :

7 Médicaments inscrits sur la liste I: spécialités présentant les risques les plus élevés. Médicaments inscrits sur la liste II : substances vénéneuses considérées comme moins dangereuses.

8 Article 8 de la Loi SR : "Le personnel intervenant dans le domaine de la santé de la reproduction est tenu de se soumettre aux normes de compétence, aux protocoles de services et aux règles de déontologie afférentes à sa profession ou à son activité. Les normes de compétence en santé de la reproduction, relatives à chaque catégorie professionnelle sont fixées par décret. 
"Le pharmacien a été formé à l'hôpital sur les soins infirmiers, je me pose la question pourquoi dans notre formation on nous a formés en injection et on nous interdit d'injecter. Ce qui est contradictoire.... les aides-infirmiers qu'on a autorisés à injecter n'ont même pas le Bac " [Pharmacien, Guédiawaye].

"Pourtant on a reçu la formation [en injection]. Pourquoi on nous a formés pour ça et on nous interdit de faire ça ? " [Pharmacien, Guédiawaye].

"Pour les produits, moi je crois que c'est aberrant quand même, les contraceptifs d'urgence sont trois fois plus dosés et on les autorise à la vente libre. Et pour les contraceptifs les moins dosés il faut une ordonnance, il faut tout harmoniser je pense "[Pharmacien, Guédiawaye].

\section{La formation insuffisante sur les services de PF}

Dans le cadre de leur formation initiale, les pharmaciens et pharmaciens-assistants (qui sont également des pharmaciens de formation) ne sont pas spécifiquement formés sur les technologies contraceptives. À la faculté, les enseignements sont relatifs aux médicaments, en général, notamment aux indications, contre-indications, posologies et effets secondaires, etc. Tout de même, dans l'exercice de leur fonction, ils sont invités à des enseignements postuniversitaires (EPU) organisés la plupart du temps par des laboratoires pharmaceutiques pour la présentation de nouveaux produits, y compris les contraceptifs. Il en est de même, des formations organisées par des structures comme l'ADEMAS lorsqu'il y a de nouveaux produits du marketing social à introduire. Par ailleurs, dans le cadre d'un programme, ADEMAS a renforcé les capacités du personnel de pharmacies réparties dans 11 régions du Sénégal en PF. Cependant ces formations étaient limitées au renforcement des connaissances des pharmaciens et agents de comptoir sur la SR en général, les différentes méthodes de contraception et les effets secondaires ou situations nécessitant une référence.

En outre, seuls quelques pharmaciens ont reçu une formation en counseling à l'occasion de leur participation à des expériences pilotes ${ }^{9}$. Un pharmacien de Dakar souligne : "On n'a pas la formation à la base. C'est ça qui nous manque en fait. On n'a pas une bonne base qui nous permette vraiment d'être imprégnés comme il faut ". Un autre renchérit en déclarant que : "On n'a pas été formé pour ça, c'est l'outil qui fait défaut ".

Pour le reste du personnel des pharmacies privées, communément dénommés vendeurs ou agents de comptoir, ils n'ont généralement pas une qualification spécifique. Hormis quelques-uns issus des écoles de formation de délégués médicaux, de sages-femmes ou d'infirmiers, ils ont pour la plupart reçu une formation sur le tas, dispensée par le pharmacien lui-même et limitée le plus souvent à la connaissance des règles de délivrance des produits. Les autres informations qu'ils reçoivent le sont souvent à l'occasion de visites des délégués médicaux lors de la présentation de nouveaux produits de PF mis sur le marché.

9 Par exemple, Intrahealth a travaillé avec l'Ordre des Pharmaciens pour la formation au "Paquet Intégré de Services de Qualité " avec un accent sur la PF. De même dans le cadre du projet ISSU, MSI a travaillé avec un réseau de 82 pharmacies pilotes pour les former au counseling, au marketing et à la référence des clientes auprès des structures habilitées. 


\section{L'inadéquation des locaux}

La majorité des personnes interrogées, aussi bien les parties prenantes de la PF que les pharmaciens, soulignent l'inadéquation de l'infrastructure des pharmacies privées. En effet, la plupart des pharmacies sont confrontées à un problème d'espace. Elles sont souvent étroites et les client(e)s sont généralement reçu(e)s au comptoir. Certes de nombreuses pharmacies disposent à l'arrière d'une salle qui permet de recevoir les client(e)s en privé, au besoin, mais cet espace, qui est en réalité le bureau du pharmacien est également souvent très réduit. Et même dans les cas où la pharmacie dispose d'assez d'espace, la configuration des locaux ne permet pas toujours de garantir la confidentialité qui devrait accompagner l'offre de services de PF. Un pharmacien de Dakar témoigne : "On n'a pas l'espace que nous voulons. Qui parle de PF, parle quand même de confidentialité. Donc il faut un coin déjà pour parler avec la personne, il faut un coin effectivement pour faire ces injections, pour placer le Jadelle. II faut un espace déjà bien aménagé, propre où on peut faire ce genre de chose. Quand on échange avec un client, que ce soit sur la PF ou autre chose, il faut toujours quand même cette confidentialité. Donc souvent il y a des pharmaciens que nous rencontrons qui n'ont pas cet espace et là c'est un peu compliqué ".

\section{La non-disponibilité des produits}

Selon les pharmaciens, hormis le fait que les pharmacies privées ne disposent pas de toute la gamme de méthodes contraceptives offertes par le Programme PF, elles font aussi quelque fois face à des ruptures de stocks de certaines marques de CO. Cette situation limite la disponibilité des produits. Les ruptures de stocks ${ }^{10}$ peuvent être dues essentiellement à l'arrêt de fabrication du produit ou à la délocalisation d'un laboratoire d'un pays à un autre. Ces ruptures se seraient accentuées durant les six (6) derniers mois précédant cette étude. Selon certaines sources, ces ruptures sont devenues récurrentes depuis que l'un de leurs principaux fournisseurs (Pfizer) n'est plus sur place pour assurer la fourniture de plusieurs marques de pilules que les populations sénégalaises avaient l'habitude d'utiliser. Ainsi, au niveau du secteur privé, en dehors du Sécuril distribué par des grossistes privés via le marketing social, il y aurait une rupture totale des autres marques de $\mathrm{CO}$ sur l'ensemble du territoire.

\section{Les convictions religieuses}

Même si aucun des répondants n'a déclaré avoir une contrainte personnelle de nature religieuse à l'offre de services de PF, ils reconnaissent que certains membres de la profession en ont. Cela pourrait constituer un obstacle à l'offre de services de PF au niveau des pharmacies privées. Un pharmacien de Dakar déclare en effet : "Il y a aussi quelques pharmaciens réticents pour des convictions religieuses qui ne vendent pas de préservatifs, qui ne vendent pas de contraception orale, qui ne veulent même pas en entendre parler. Lors du tri pour les pharmacies pilotes, il y a eu des cas comme ça qui ont été rencontrés et qui ont dit qu'ils ne sont pas dans ce jeu. Ils étaient catégoriques et rien ne leur fera changer d'avis ".

10 La rupture de stock d'une méthode est mesurée comme étant la non-disponibilité de la méthode pendant au moins 24 heures : i) au cours des 30 derniers jours et ii) au cours des 12 derniers mois. 


\section{Perceptions de l'implication des pharmacies privées dans l'offre de services de PF}

L'implication insuffisante des pharmacies privées dans l'offre de services de PF a fortement été déplorée, surtout par les pharmaciens eux-mêmes. Ils pensent, tout comme la plupart des parties prenantes, qu'ils peuvent jouer un rôle beaucoup plus actif, notamment dans l'offre initiale de produits contraceptifs. Seuls quelques interviewés pensent que le rôle du pharmacien doit se limiter à la délivrance des médicaments, aux conseils et à la référence, essentiellement pour ne pas empiéter sur les prérogatives du personnel médical. De fait, presque toutes les personnes interrogées ont manifesté un grand intérêt à l'idée de mieux impliquer les pharmacies privées dans l'offre de services de PF. Qui plus est, beaucoup de pharmaciens se disent investis d'une mission de santé publique et sont ouverts à cette idée. Ils seraient même " demandeurs " selon un représen-

\section{Une franche collaboration} entre le secteur public et le secteur privé peut aboutir à de meilleurs résultats. tant du gouvernement. Cet avis est corroboré par les propos suivants :

"Voilà, nous c'est ce que nous demandons avec la DSRSE. On en a discuté, et $X[. .$.$] nous avait promis$ qu'ils allaient eux-mêmes faire le plaidoyer pour qu'on change les textes.... Une révision des textes que nous avions demandée depuis, nous en tant que syndicat...C'est des textes de 1953, obsolètes. Tout ce qui est obsolète on le jette. Tout ce qui doit être revu contextuellement, que ça soit bien revu et ce qui peut être corrigé que tout soit revu en tout cas... parce que quand même le rôle du pharmacien a changé, il a beaucoup évolué " [Pharmacienne, Dakar].

"Je crois au-delà des aspects législatifs, il faut peut-être que les pharmaciens prennent les devants. C'est-à-dire au lieu d'attendre que l'on nous autorise, qu'on aille peut-être au niveau de l'autorité médicale, du Ministre de la santé par le biais de l'Ordre des Pharmaciens, du syndicat, véritablement pour donner notre volonté de participer effectivement dans la prise en charge des programmes de PF " [Pharmacien, Pikine].

"En tout cas moi, à toutes les instances auxquelles j'ai participé où j'ai travaillé avec eux [les pharmaciens], c'était des cris de cœur. Tous, ils veulent faire l'offre de services. Tous les pharmaciens veulent le faire. Et en plus de ça, ils se voient dévalorisés par le fait de ne pas pouvoir offrir ne serait-ce qu'une injection " [Représentant ONG].

Plusieurs arguments en faveur de cette implication ont été avancés :

\section{Cela pourra contribuer à améliorer les programmes SR et PF.}

Pour beaucoup de personnes interrogées, l'implication des pharmaciens dans l'offre de services de PF, pourrait non seulement élargir les acteurs de l'offre, mais surtout accroître les résultats/performances du Programme de SR en général et de la PF en particulier grâce à l'intégration de leurs données dans le système d'information sanitaire. De plus, améliorer la couverture PF pourrait permettre de réduire les BNS, et par conséquent de contribuer à 
la hausse du TPCm et à la baisse de la mortalité maternelle et infantile. En définitive, cela aiderait à atteindre les objectifs nationaux et internationaux. En réalité, certains pensent que les données capitalisées en matière de PF seraient sous-estimées car elles ne prennent pas en compte les données des pharmacies privées.

\section{Cela pourrait renforcer le partenariat public-privé (PPP).}

Certaines parties prenantes de la PF notent une meilleure prise en compte du secteur privé dans l'élaboration et la mise en œuvre des politiques de santé, grâce notamment à la mise en place de "l'Alliance du secteur privé de la santé ". Mais pour elles, l'implication des pharmacies privées à l'offre de services de PF constituerait une étape supplémentaire de l'appui du secteur privé aux politiques de santé du ministère. Cela pourrait ainsi mieux renforcer le PPP, y compris par la prise en compte des données de ce secteur.

\section{Les pharmaciens ont reçu une formation en soins infirmiers.}

Au cours de leur formation universitaire, les pharmaciens sont tenus de faire entre la quatrième et la cinquième année, un stage de six (6) mois en soins infirmiers, en milieu hospitalier. Cette formation leur permet d'acquérir les compétences nécessaires pour pouvoir effectuer, entre autres, des injections.

\section{Certains pharmaciens offrent déjà des méthodes contraceptives de manière officieuse.}

Sur le plan opérationnel, malgré l'existence de barrières juridiques, nombreux sont les interviewés qui pensent que l'offre de services de PF dans les pharmacies privées est courante. Cela est confirmé par certains pharmaciens qui reconnaissent prescrire occasionnellement l'offre initiale de méthodes de contraception, le plus souvent lorsque les clientes font face à une urgence (exemple : arrivée impromptue du mari migrant).

"Qu'on le veuille ou pas, les pharmaciens font des injections [de méthodes contraceptives]. Les vendeurs font des injections. Donc, je crois que pour faciliter la chose, si on les forme, on les recycle, on fait un suivi, ça va faciliter les choses " [Représentant ONG].

"C'est aux infirmiers ou sages-femmes de faire des injections. Mais dans certains cas exceptionnels, extrêmes, on est obligé d'intervenir, de faire des injections, des Depo par exemple qui sont une forme injectable de contraception " [Pharmacienne, Pikine].

\section{Certaines méthodes de PF sont offertes par des agents de santé moins qualifiés.}

Beaucoup de personnes interrogées pensent que les pharmaciens, qui ont un niveau d'éducation beaucoup plus élevé, devraient offrir les services de PF puisqu'aujourd'hui au Sénégal certains personnels moins qualifiés, tels que les personnels communautaires, y compris les matrones et agents de santé communautaire (ASC) sont capables et autorisés à faire l'offre initiale de pilules et d'injectables. 
"C'est ce que nous revendiquons, que si une matrone peut au fin fond du Sénégal faire une injection, le pharmacien est aussi outillé pour le faire " [Pharmacien, Pikine].

"A mon avis c'est possible, simplement comme j'ai eu à le dire au cours d'une réunion, aujourd'hui il y a des agents de santé avec qui quand même nous partageons le métier mais qui ont une formation moindre, en tout cas moins importante que celle du pharmacien, je veux parler des aides, je veux parler des matrones qui offrent des services que nous ne pouvons pas offrir " [Pharmacien, Dakar].

"Le relais communautaire, il n'est pas un personnel médical. II est un personnel communautaire, pourtant maintenant tout le monde est convaincu qu'ils peuvent offrir des pilules et même des injectables au niveau communautaire. Mais, à plus forte raison un docteur en pharmacie " [Représentant ONG].

6. Cela pourrait répondre aux besoins spécifiques de certaines populations en matière de contraception, notamment les femmes intellectuelles, les veuves, les divorcées, les jeunes (pour la CU notamment), les hommes (surtout pour les condoms), etc.

Pour de nombreux interviewés, surtout parmi les pharmaciens, la satisfaction des besoins en information et/ou contraception, de façon discrète et rapide, et auprès d'une personne familière et de confiance, pourrait encourager les populations à se diriger vers les pharmacies privées pour des services de PF. De plus, sur le plan géographique et financier, avec le rapprochement du service, la méthode serait plus accessible, sans coûts de transport ni paiement de ticket modérateur.

7. Les pharmaciens peuvent aider à convaincre les maris compte tenu de leurs relations avec les populations.

Certains pharmaciens pensent qu'au même titre que d'autres leaders communautaires, ils peuvent aider à convaincre certains maris réticents à la PF. En effet, au-delà de leur rôle de conseiller, beaucoup ont parfois eu à jouer un rôle de médiateur pour certains couples, du fait de leur proximité avec les populations avec lesquelles ils entretiennent souvent des relations de confiance.

"Certaines clientes veulent faire la PF, mais leurs maris n'acceptent pas. Des fois elles viennent accompagnées de leurs maris pour voir le pharmacien qui leur explique tout. Beaucoup d'hommes ne comprennent pas, mais le pharmacien peut les convaincre.... le pharmacien qui est un voisin et qui a des relations particulières de bon voisinage peut les appeler pour les sensibiliser. On peut leur rendre beaucoup de services "[Pharmacien, Guédiawaye].

\section{Personnel à impliquer dans l'offre de services de PF}

Les avis sont partagés sur le personnel des pharmacies privées à impliquer dans l'offre de service de PF. Si une partie des personnes interrogées pense que seul le pharmacien titulaire ou son assistant doivent être impliqués, car étant le seul personnel qualifié, la majorité 
pense, au contraire, que certains vendeurs/agents de comptoir expérimentés peuvent l'être aussi. Ils soulignent que ces agents, qu'ils assimilent à du personnel communautaire, s'ils sont bien formés, peuvent également offrir des services de PF, et cela pour plusieurs raisons : ils constituent souvent la majorité du personnel des pharmacies privées ; les pharmaciens titulaires sont généralement absents ; ce sont les vendeurs qui sont le plus souvent en contact avec les populations ; et la plupart des vendeurs bénéficient d'une longue expérience leur permettant de faire face à beaucoup de situations.

"Tout le personnel de la pharmacie devrait être impliqué à offrir ces services, et même les vendeurs. Le pharmacien n'est pas tout le temps sur place et certaines pharmacies n'ont pas d'assistants. Certains vendeurs sont rôdés avec une bonne expérience dans la vente de médicaments. S'ils sont bien formés, ils pourront assurer ces services " [Pharmacien, Dakar].

"Les vendeurs aussi, parce que si vous regardez dans les officines les deux tiers du personnel c'est des vendeurs. II y a un pharmacien et peut être un assistant. Donc, on gagnerait à former ces vendeurs-là. Parce que ce sont ces vendeurs qui sont en contact avec les populations " [Distributeur pharmaceutique].

"Le pharmacien aussi n'est pas toujours disponible, il n'est pas toujours au niveau de la pharmacie. Le plus souvent quand les gens viennent, ils ont à faire à l'agent de comptoir ou bien à l'assistant du pharmacien " [Représentant du gouvernement].

"Le personnel qui est là n'est pas un personnel qualifié. Pour moi, c'est comme un ASC qui est là.Donc, c'est pourquoi je dis c'est la même règle qui régit l'ASC qui devrait être appliquée à cet agent qui est à côté du pharmacien " [Représentant du gouvernement].

\section{Services de PF susceptibles d'être offerts par les pharmaciens}

Les interviewés pensent de façon quasi-unanime qu'en plus du rôle de conseiller qu'ils jouent déjà, les pharmaciens privés pourraient jouer un rôle plus important en matière de PF. Ils pourraient faire le counseling pour permettre à la femme de connaître l'ensemble des méthodes contraceptives disponibles au niveau du Programme National afin de pouvoir faire un choix éclairé. Cependant, la grande majorité des répondants pense que les pharmaciens pourraient aller encore plus loin et être impliqués dans l'offre initiale de méthodes de courte durée telles que les $\mathrm{CO}$ ou les injectables. Selon eux, ce service est largement à leur portée. De plus, les répondants pensent même qu'après l'offre de méthodes, les pharmaciens privés devraient faire le suivi des clientes (gestion des rendez-vous, renouvellement régulier) et gérer les effets secondaires.

Par contre, concernant l'offre de méthodes de longue durée, telles que les implants ou le DIU, la plupart estime que cela nécessite des compétences particulières et un acte médical avec un équipement adéquat qui n'existe pratiquement pas dans les pharmacies privées. Elles pensent donc que cette offre doit être réservée aux structures de santé. 
"Ce qu'on peut faire c'est juste les injections, la voie orale. C'est ça. Pour les implants et tout le reste, il faut couper, inciser, placer le produit, il y a le contact avec le sang. II faut que la femme soit dans une certaine position qui n'est pas recommandée en pharmacie. Donc il y a tout cela qui joue " [Pharmacien, Pikine].

\section{Opportunités pour l'implication des pharmacies privées dans l'offre de services de PF}

L'implication des pharmacies privées dans l'offre de services de PF est considérée par les personnes interrogées comme une stratégie intéressante du fait des nombreuses opportunités, notamment :

\section{Les pharmacies constituent souvent le premier recours aux soins des populations.}

La quasi-totalité des personnes interrogées soutient que pour la majorité des populations, surtout de la banlieue, les pharmacies constituent la porte d'entrée au système de santé du fait de leur proximité. En cas de souci en matière de santé, le premier réflexe est de se rendre à la pharmacie.

"La pharmacie constitue aujourd'hui le premier recours, la porte d'entrée du système de santé. Les gens ont l'habitude de venir à la pharmacie chaque fois qu'ils ont un petit problème, et je crois que c'est une excellente opportunité de pouvoir donner accès à ces méthodes de contraception aux populations " [Pharmacien, Dakar].

"Les pharmacies sont une porte d'entrée pour tout. Je ne dirai pas pour la PF seulement, mais pour tous les autres services. Les populations préfèrent aller à la pharmacie. J'ai mal à la tête, je vais à la pharmacie. J'ai mal au ventre, je vais à la pharmacie "[Représentante du gouvernement].

"Surtout en banlieue les gens ne vont jamais à l'hôpital. C'est plus facile d'aller en pharmacie qu'à l'hôpital. C'est ça la mentalité des banlieusards " [Pharmacien, Pikine].

\section{Les pharmacies privées disposent déjà d'une expérience dans la dispensation/vente de produits contraceptifs.}

Comme l'a montré la revue de la littérature, presque toutes les pharmacies visitées vendent des produits contraceptifs depuis leur ouverture, même si la plupart ne dispose pas de l'ensemble des méthodes. Cette expérience pourrait être mise à profit pour une implication effective des pharmacies privées dans l'offre de services de PF.

\section{Le nombre important des pharmacies privées.}

Beaucoup de personnes interrogées pensent qu'à un moment où l'insuffisance des structures de santé publique constitue encore un défi du système de santé, les nombreuses 
pharmacies privées ${ }^{11}$ existantes pourraient constituer des sources additionnelles d'offre de services de PF. De plus, elles sont géographiquement mieux réparties que les structures de santé publique et offrent ainsi une meilleure accessibilité.

"Les pharmacies privées c'est ce qu'il y a de mieux réparti sur le territoire sénégalais. II y a des coins où il n'y a pas de poste de santé, mais où il y a un pharmacien qui est là-bas et qui va jouer plus que son rôle de pharmacien.... Donc avec cette répartition, ce maillage sur le territoire, les pharmaciens peuvent apporter beaucoup " [Pharmacien, Dakar].

"Du point de vu potentiel, quand on a 1400, 1500 pharmacies sur le territoire national c'est autant de points de prestation qu'on aurait pu avoir, c'est des opportunités manquées quoi " [Représentant du gouvernement].

\section{La disponibilité du personnel par rapport aux horaires d'ouverture}

Les pharmacies privées ont des horaires d'ouverture plus longues que les structures de santé publique. En effet, la plupart ouvrent à $8 \mathrm{~h}$ et vont au-delà de $22 \mathrm{~h}$. Certaines fonctionnent même $24 \mathrm{~h} / 24$, en s'appuyant généralement sur une (1) équipe du matin et une (1) du soir. Cela permet aux client(e)s d'y trouver du personnel au moment qui leur convient et de pouvoir ainsi vaquer tranquillement à d'autres occupations.

"En tout cas on peut trouver une pharmacie ouverte de $8 \mathrm{~h}$ à $23 \mathrm{~h}$, voire minuit et ensuite il y a les pharmacies de garde. Donc au moins cette plage horaire-là, on ne l'a pas dans les structures publiques " [Pharmacien, Dakar].

"C'est ouvert de jour comme de nuit pratiquement, c'est ouvert même les jours fériés ou en tout cas il y a un service de garde. Donc ils peuvent aller et quand ils ont un premier besoin, même un mal de tête, on pense d'abord aller à l'officine et une femme qui doit aller au marché peut passer là-bas rapidement prendre sa méthode et puis s'en aller continuer son travail. Ce qui est différent des structures sanitaires " [Représentant du gouvernement].

\section{L'intérêt manifesté par les pharmaciens et autres acteurs clés de la PF à l'idée de voir les pharmacies privées jouer un rôle plus important dans ce domaine.}

La majorité des personnes interrogées pense que le fait que la quasi-totalité des pharmaciens et autres acteurs clés de la PF adhèrent à l'idée de mieux impliquer les pharmacies privées dans l'offre de services de PF est un atout à capitaliser, en témoignent les propos de ce représentant du gouvernement : "Je pense qu'il y a parmi eux des champions...qui comprennent bien les enjeux de la PF et qui sont prêts à accompagner. Je pense aussi que le fait qu'ils soient là à chaque fois qu'on les invite dans des réunions, qu'ils prennent la parole, qu'ils disent ce qu'ils en pensent, ça signifie que c'est un intérêt qu'ils trouvent dans ça, ils pensent qu'ils peuvent être utiles à la population. Donc ça c'est un potentiel sur lequel on peut construire quelque chose".

11 En Juillet 2016, une estimation indique qu'il y aurait entre 1300 et 1500 pharmacies privées au Sénégal. 


\section{Défis liés à l'implication des pharmacies privées}

Les informations recueillies ont permis d'identifier certains défis qui pèsent sur l'offre de services de PF par les pharmacies privées. Il s'agit notamment de :

\section{La révision des textes}

Si les pharmaciens doivent jouer un rôle plus important en matière de PF, il faudrait en priorité que le cadre juridique soit révisé afin de lever les restrictions et ainsi les protéger. Selon eux, cela passe nécessairement par une réelle volonté politique. Le MSAS à travers la Division du partenariat public-privé a mis en place un cadre de dialogue et commandité le recensement des textes qui régissent le secteur privé. Cependant, malgré les efforts fournis pour l'identification des dispositions qui posent

Un pharmacien bien formé peut offrir une méthode comme tous les autres agents de santé, mais sous réserve que la législation le lui permette. problème, aucune avancée n'a été notée lors de l'examen en cours des textes proposés ${ }^{12}$ pour les pharmacies lors de l'atelier de révision du cadre juridique et réglementaire du secteur privé de la santé. Par ailleurs, des propositions allant dans le sens d'intégrer l'offre de service de PF dans les missions du pharmacien ont été formulées par les représentants du secteur. Le défi consiste donc, selon ces derniers à faire accepter ces nouvelles propositions qui favoriseraient véritablement l'implication des pharmaciens dans l'offre de services de PF. En outre, il faudra revoir l'ensemble des politiques, normes et protocoles (PNP) ${ }^{13}$ affectés par cette révision.

"Il y a des actes à prendre, notamment il y a des lois, il y a des règlements à modifier pour permettre au pharmacien de faire certaines choses parce que [...] le pharmacien il est dans le privé, et donc il assume seul sa responsabilité, alors que lorsqu'on est dans le public on est quand-même couvert sur certaines choses par l'Etat parce qu'on est agent de l'Etat " [Représentant Société Civile].

\section{La formation}

En dehors des aspects législatifs, la formation constitue pour certaines personnes interrogées, notamment parmi les pharmaciens, le plus gros défi. D'une part, il faudra assurer la formation sur le tas de tous les pharmaciens privés déjà homologués. Cependant, même si le MSAS dispose d'un pool de formateurs dans chaque région du Sénégal, plusieurs répondants pensent qu'organiser ces formations ne sera pas une chose aisée compte tenu de la difficulté à mobiliser le personnel des pharmacies durant quelques jours, par crainte de leur part de perte de recettes. D'autre part, l'offre de services de PF devra être intégrée

12 En janvier 2017, un atelier de relecture et de réécriture des textes proposés pour la révision du cadre juridique et règlementaire du secteur privé de la santé a été organisé par le MSAS à travers la Division du partenariat publicprivé, à l'issue duquel des ateliers de finalisation et de validation des textes sont prévus.

13 Les PNP sont revus tous les 4 ans environ. La dernière révision des PNP relatifs à la SR/PF a été faite en 2016. 
dans les curricula de formation initiale, de sorte que les nouveaux sortants soient dotés des capacités à la base. Cependant, cette procédure peut s'avérer lente et compliquée. Par ailleurs, les répondants proposent que les pharmacies soient formées sur le counseling, les technologies contraceptives, la gestion des effets secondaires, et aussi sur les PNP et les techniques de communication. De plus, pour garantir la qualité des services, certains pensent qu'il faudra également aborder des aspects tels que l'assurance de la qualité. Concernant la collecte des données, une formation sur le remplissage des outils de gestion sera indispensable.

À l'unanimité, les interviewés pensent que la formation sur le tas devra être assurée par le MSAS à travers la DSRSE et les districts sanitaires, avec l'implication du CNOPS et du syndicat des pharmaciens, et l'appui des partenaires techniques et financiers. Le défi réside donc dans la mise en œuvre effective de ces formations.

\section{La disponibilité/approvisionnement des produits}

Concernant la disponibilité des produits, les défis se posent à plusieurs niveaux. D'abord, certaines parties prenantes de la PF se posent la question de savoir si la disponibilité de la gamme complète des contraceptifs du Programme PF sera assurée au niveau du secteur privé ou s'il faut définir une autre gamme pour ce secteur? Ils se demandent également ce que sera la nature des produits qui y seront distribués : s'agira-t-il de produits du secteur public (souvent des génériques) ou privé (surtout des spécialités) ? Beaucoup de personnes interrogées, surtout les parties prenantes de la $\mathrm{PF}$, pensent que la réponse à ces questions dépendra fortement du circuit d'approvisionnement qui sera retenu pour les pharmacies privées. Certains croient que le circuit d'approvisionnement actuel à travers les grossistes privés reste incontournable. D'autres pensent que pour améliorer l'accès des populations aux produits contraceptifs et ainsi palier aux ruptures, il faudrait plutôt mettre l'accent sur un circuit parallèle via la Pharmacie nationale d'approvisionnement (PNA). Cela pourrait se faire par exemple dans le cadre du programme Reproductive, Maternal, Newborn and Child Health/Santé de la reproduction et survie de l'enfant ${ }^{14}$ qui a mis en place un plan de sécurisation de 17 produits d'importance vitale pour la santé de la mère et de l'enfant, dont font partie les produits PF. II s'agira donc de voir comment rendre disponibles ces produits au niveau des pharmacies. Pour d'autres encore, le "Informed Push Mode ${ }^{15}$ " présente également une alternative assez intéressante.

14 II s'agit du programme " Reproductive, Maternal, Newborn and Child Health/Santé de la reproduction et survie de l'enfant " initié par la Commission des Nations Unies sur les produits essentiels pour la survie des femmes et des enfants, et qui vise l'amélioration de l'accès et de l'utilisation appropriée des médicaments, équipements médicaux et fournitures ou des produits de santé essentiels.

15 L'IPM est un modèle de distribution de type "Push " (système de réception passive sans commande), auquel des opérateurs logistiques privés ont été associés. Ce modèle de distribution rapproche la source de l'approvisionnement (le transporteur) de la source de la demande (les clientes des structures de santé) et simplifie les étapes intermédiaires. II repose sur plusieurs points clés : le stock initial de contraceptifs est fourni gratuitement à chaque point de prestation de service ; des logisticiens privés sont chargés du réapprovisionnement des points de prestation de service mensuellement afin de maintenir un niveau de stock minimum suffisant ; le point de prestation de service ne paie que pour les produits qui ont été vendus et conserve la marge réalisée; les logisticiens collectent les données de consommation de produits au moment de leur livraison et les renvoient au médecin chef du district. Le développement de ce modèle d'approvisionnement a permis de réduire considérablement les ruptures de stock. 
Un autre défi concerne les produits offerts à travers le marketing social. À ce jour, ils sont exclusivement financés par les partenaires financiers. Comment l'État s'impliquera-t-il pour en assurer la pérennité ?

\section{Le réaménagement des pharmacies privées}

Selon la politique pharmaceutique, l'aménagement d'un espace de confidentialité dans la pharmacie est obligatoire. Toutefois, certains pharmaciens reconnaissent que même si la majorité en dispose, quelques-uns n'en ont pas encore. Et même pour ceux qui en ont, ces espaces restent parfois limités et servent de bureau pour le pharmacien. Dans tous les cas, des réaménagements s'imposeront afin d'avoir un local approprié pour garantir la confidentialité sensée accompagner l'offre de services de PF et permettre une interaction efficace. Pour certains, ces aménagements pourraient être difficiles car la plupart des pharmaciens ne sont pas propriétaires de leurs locaux. Ils devront obtenir l'autorisation des bailleurs, ce qui peut s'avérer compliqué. L'introduction de nouveaux services pourrait aussi occasionner des dépenses supplémentaires onéreuses. Comment faire pour que ces rénovations et coûts ne soient pas des obstacles à la volonté des pharmaciens de s'impliquer dans l'offre de services de PF ? Comment ces dépenses seront-elles absorbées par les pharmaciens?

\section{La collecte des données et leur transmission}

Si les pharmacies étaient autorisées à offrir des services de PF, elles devraient désormais transmettre leurs données statistiques au district sanitaire dont elles relèvent pour être prises en compte dans le Système National d'Information Sanitaire. De plus, plusieurs parties prenantes clés ont attiré l'attention sur la faiblesse du secteur privé dans le recueil de données statistiques. Cela a d'ailleurs été constaté lors du remplissage du formulaire de collecte sur le counseling et les ventes de produits de PF. En effet, en dehors de l'enregistrement des statistiques d'entrées et sorties de produits dans le cahier des ventes ou dans un logiciel informatique, les données sur le profil des client(e)s en général, encore moins sur ceux/celles ayant reçu des conseils sur la PF, ne sont pas enregistrées. Les pharmaciens interrogés ont expliqué que non seulement ils n'ont pas les outils nécessaires pour le faire, mais que jusqu'ici ils n'en ont pas ressenti le besoin puisque le district ne leur en a jamais exprimé la demande. Par ailleurs, certains pharmaciens ayant participé à des expériences pilotes ont déclaré avoir observé des irrégularités dans la collecte des données sur le counseling et les références effectuées, bien que des outils aient été mis à leur disposition.

Les avis sont partagés sur la manière de collecter les données statistiques des pharmaciens privés : plusieurs parties prenantes, notamment les représentants du gouvernement, pensent que l'idéal serait qu'ils utilisent les mêmes registres que le secteur public pour en faciliter l'exploitation (et la formation des pharmaciens), tandis que d'autres avancent que, vu le temps que nécessitera le remplissage de ces registres, les autorités sanitaires devraient plutôt prévoir des outils plus simples pour garantir une bonne complétude et promptitude des rapports mensuels, ainsi qu'une bonne remontée des données vers les districts.

Le défi est donc de faire en sorte que le secteur privé participe activement au SNIS et four- 
nisse des données de qualité et à temps pour contribuer à une estimation plus juste des données de routine.

\section{Le suivi/supervision des pharmacies}

Comme toute délégation de tâches, l'offre de services de PF par les pharmacies doit, selon certains, être suivie et supervisée conformément aux PNP, pour assurer la qualité des services.

À cet effet, certaines parties prenantes de la PF pensent que le suivi et la supervision des activités des pharmacies devront être intégrés dans la supervision de routine au niveau des districts, ce qui constitue un gros défi logistique et organisationnel au regard des irrégularités encore constatées dans la supervision des structures publiques.

\section{Le coût des services}

Beaucoup de pharmaciens ne considèrent pas le coût comme un défi. Ils disent que non seulement les client(e)s seront libéré(e)s des frais de transport, mais qu'en plus, n'ayant pas à acheter un ticket modérateur comme à l'entrée de la structure de santé publique, ils/elles ne paieront que le prix du produit. Ils jugent également que les produits du marketing social qu'ils vendent sont abordables. Un pharmacien de Guédiawaye déclare : "Moi personnellement je ne le considère pas comme un problème. C'est exceptionnel qu'une dame nous dise "vous n'avez pas moins chers ? "Aujourd'hui les pilules sont relativement accessibles. Pour le cas du Sécuril, il est à 400F, pour les autres il y en a qui sont à moins de 1000F ou à 1500F. Je pense que le coût ne pose pas de problème ".

De plus, ils font remarquer qu'ils ont leur clientèle et que quel que soit le prix, beaucoup de client(e)s préfèrent se rendre dans les pharmacies où ils/elles se sentent plus à l'aise. Cela est confirmé d'ailleurs par un partenaire au développement qui souligne que "Elles [les pharmacies] ont leur clientèle. Ça veut dire que certainement c'est un choix.... C'est une question de choix ; ça élargit le choix de la clientèle, des populations. Quand ils sauront que ça existe, et chez le privé et chez le public, ils verront par eux-mêmes là où ils sont beaucoup plus à l'aise.Parce que ce n'est pas une question d'offrir juste des services, c'est une question également de choix. Comme on dit, il faut que la personne puisse choisir. Donc, ça c'est élargir au moins le champ, élargir la couverture et élargir également le choix, la possibilité de choisir pour la personne".

Ces propos sont corroborés par ceux d'une représentante du gouvernement qui s'appuie, elle, sur la nécessité de se conformer à l'approche du marché total. Elle souligne que : "II ne faut pas aussi les forcer [les pharmacies] coûte que coûte à ce qu'elles ne vendent que des génériques. II y a une certaine population qui est à l'aise, qui, même si le produit vaut 2000f, elle a la possibilité d'acheter. Donc laisser aussi à l'officine cette possibilité. Celui qui veut vendre la spécialité qu'il mette en place la spécialité, mais aussi le médicament générique. Et c'est à la cliente qui viendra, par rapport à sa capacité et son désir parce qu'il y a certaines populations qui disent que les produits qui ne coûtent pas cher ne sont pas 
de qualité aussi. Donc il faut vraiment l'approche de marché total aussi et ne pas vouloir rendre tout gratuit ".

Par contre, pour la majorité des parties prenantes de la PF, la diversité des prix dans les pharmaciens privés est un défi majeur. Il faudra nécessairement les harmoniser. Selon elles, si les pharmaciens étaient autorisés à offrir des méthodes de PF, l'État devrait baisser les coûts afin que les prix proposés soient similaires à ceux du secteur public pour rendre les produits vraiment accessibles et pour ne pas décourager certain(e)s client(e)s. Cela sous-entend des subventions de l'État. Certains n'excluent pas aussi la possibilité de revoir la nature des produits contraceptifs distribués dans les pharmacies pour la mise à disposition de génériques, comme le soutient cette représentante d'ONG : "Il y aura à voir dans quelle mesure on peut appliquer la même chose qui a été fait avec les médicaments essentiels. Voilà les génériques qui sont en pharmacie et qui coûtent moins cher que les produits de spécialité. Bon, si on peut faire la même chose avec les produits contraceptifs certainement ça peut être moins cher au niveau des pharmacies et puis l'accessibilité serait plus importante".

Dans tous les cas, le défi est que les services soient offerts aux client(e)s à des prix abordables, tout en permettant aux pharmaciens privés d'avoir des marges suffisantes pour les motiver.

\section{La coordination entre les districts sanitaires et les pharmacies privées}

De nombreux pharmaciens ont dénoncé l'attitude des districts sanitaires qu'ils jugent négative vis-à-vis d'eux. Selon eux, les autorités des districts ne les considèreraient pas comme faisant partie intégrante du système de santé. De ce fait, ils se sentent laissés pour compte alors même que sur le terrain, il devrait y avoir un lien entre les structures sanitaires de base (centres de santé et postes de santé) et les pharmacies privées. Pire, certains districts les considèreraient comme des rivales. Cette situation ne faciliterait pas leur prise en compte dans les nombreuses interventions initiées dans les districts, et ne favoriserait pas leur implication dans les activités du secteur public, notamment le Programme PF.

De l'avis de certaines parties prenantes de la PF, cette absence ou insuffisance de coordination entre les pharmacies et les médecins-chefs et/ou autres personnels de districts n'est pas simplement le fait du district, mais aussi des pharmaciens eux-mêmes qui ne voudraient pas remplir les registres pour la production des données statistiques.

Le défi est donc de créer la confiance entre les deux (2) parties pour une collaboration harmonieuse, d'où la nécessité d'une bonne sensibilisation sur les rôles et responsabilités de chacun.

"Le district ne considère pas les pharmaciens comme faisant partie des agents de santé de la zone et ça ce n'est pas bon " [Pharmacien, Pikine].

"Mais les districts là, ils ne savent même pas que les pharmacies existent dans leur district. C'est comme s'ils les écartaient d'office et ça ce n'est pas normal "[Pharmacien, Guédiawaye]. 


\section{La gestion du temps dans les pharmacies}

Les pharmacies font partie du secteur privé à but lucratif, et pour ces acteurs généralement "le temps c'est de l'argent ". Ainsi, pour certaines parties prenantes, elles pourraient se plaindre de la perte de temps liée à la prise en charge d'une cliente de PF et à l'élaboration des rapports, surtout si elles ne disposent pas d'assez de personnel ou si le prix de cession des produits ne s'avère pas assez profitable pour elles.

Certaines parties prenantes sont même sceptiques de les voir offrir les services selon les PNP et pensent que certaines pourraient être tentées de faire un counseling orienté. De même, leur participation aux réunions de coordination au niveau des districts pourrait s'avérer contraignant.

"Le jour où ils [les pharmaciens] accepteraient de prendre la cliente comme on la prend au niveau des structures publiques, ils pourraient offrir les méthodes, parce que c'est peut-être une perte de temps pour eux et " time is money " [Membre association professionnelle].

"Il [le pharmacien] doit faire du bénéfice et il ne peut pas consacrer tout son temps à donner des méthodes contraceptives, donc ça, ça peut être un problème, voilà. " [Représentant du gouvernement]. 


\section{Discussion}

Les résultats issus de l'analyse des données qualitatives montrent des points de vue similaires sur la plupart des sujets abordés avec les deux groupes cibles.

Même si beaucoup de pharmaciens privés n'ont pas le sentiment d'être impliqués dans la $\mathrm{PF}$, il est apparu qu'en plus de la vente de produits contraceptifs, ils jouent déjà un rôle non négligeable de relais de l'information à travers les conseils prodigués aux populations dans ce domaine. Le fait pour certains projets d'avoir associé des pharmacies privées à leurs interventions pilotes renforce davantage ce rôle. Force est de constater tout de même que leur non-participation à l'offre effective de services de PF résulte des capacités limitées de leur personnel dans ce domaine, de l'existence de certaines contraintes logistiques, mais surtout d'un environnement juridique et règlementaire peu favorable à leur implication.

Par ailleurs, les résultats de la recherche qualitative interpellent sur la place du secteur privé dans le système de santé en général. À ce propos, pour aider le Gouvernement du Sénégal à honorer ses engagements internationaux et à atteindre ses objectifs nationaux, dans le contexte desquels s'inscrit le nouveau Cadre Stratégique National de la Planification Familiale 2016-2020, l'implication du secteur privé, notamment à but lucratif, dans l'offre de services de PF est impératif. Sachant que l'Organisation Mondiale de la Santé encourage une plus grande délégation des tâches au personnel non-médical, notamment au niveau communautaire, il est clair que le pharmacien a aujourd'hui un rôle plus important à jouer, un rôle qu'il joue d'ailleurs parfois en violant à l'occasion, la législation en vigueur. En étant les points de prestation de services les plus proches des populations, tel que décrit dans la carte sanitaire du Sénégal, les pharmacies présentent plusieurs opportunités à exploiter : elles constituent la première étape du circuit thérapeutique des populations; elles ont déjà une expérience avérée dans la vente de produits contraceptifs ;elles ont des horaires étendus qui permettent aux populations d'avoir accès à un personnel disponible à leur convenance ; elles ont une proximité aussi bien physique que relationnelle, ce qui les rend très attractives pour les populations ; elles sont nombreuses et largement réparties dans les quartiers, ce qui les fait apparaître ainsi comme un modèle d'offre de service qui participerait davantage à démocratiser l'offre des services de PF ; et elles pourraient contribuer à pallier la pénurie de structures et agents de santé du secteur public.

Avec 45\% d'objectif de TPCm à atteindre, l'enthousiasme des pharmaciens à contribuer aux efforts nationaux en faveur de la promotion de l'accès des populations aux services de PF est un atout considérable à capitaliser, même si l'on sait aussi que l'une de leurs missions officielles est de contribuer à la politique de santé du gouvernement. Cependant, aussi importante que soit cette motivation à s'engager dans cette dynamique, il y a des préalables à l'offre de ces services, notamment : la mise en place

Une autorisation oui, mais... une autorisation encadrée. d'un cadre juridique et réglementaire qui clarifie les services que les pharmacies privées peuvent offrir et les conditions qui y sont relatives, mais qui met aussi en place des garde-fous contre d'éventuels abus ; la formation appropriée aussi bien technique que pour la collecte des statistiques sanitaires ; le réaménagement des locaux ; la mise en place éventuelle d'un système d'approvisionnement et de logistique public et privés intégré. 
Les pharmaciens et quelques parties prenantes de la PF ne considèrent pas le coût des services comme un défi en vertu du principe de la segmentation du marché qui donne la possibilité à chacun de choisir le produit ou la structure qui lui convient selon ses moyens. Cependant, l'attention a été attirée sur la nécessité de garantir l'équité d'accès aux services dans les pharmacies privées.

Toutefois, il ne faut pas perdre de vue que les pharmaciens sont avant tout des commerciaux qui vivent des bénéfices de leurs ventes. Cette nouvelle activité d'offre de services de PF peut se révéler très exigeante (dépenses supplémentaires générées par la nécessité de réaménager leurs locaux en vue d'un meilleur cadre qui respecte les droits des clients en matière de PF, baisse des prix des produits contraceptifs, élaboration de rapports statistiques, etc.), et par conséquent susciter des interrogations chez ces acteurs sur la pertinence de leur implication. II est donc nécessaire que les pharmaciens soient sensibilisés aussi bien sur l'importance de leur contribution à la réalisation des objectifs du Programme PF et sur ce qu'ils y gagnent. En effet, même si le retour sur investissement risque de ne pas être immédiat, leur contribution à l'atteinte des objectifs nationaux et à l'amélioration de la santé des populations en tant qu'acteur du système de santé investi d'une mission de service public reste un argument de taille. De plus, ils y gagneront en termes d'harmonisation avec les normes et protocoles, de renforcement des capacités, d'encadrement et de participation à la prise de décision sur des questions relatives à la politique nationale de santé. En outre, en tant que sociétés privées, les pharmacies peuvent aussi apporter une plus-value à la promotion de la PF dans le cadre de leur responsabilité sociale. 


\section{Limites de l'étude}

Cette recherche qualitative ne peut se prévaloir d'aucune prétention d'exhaustivité. Certaines populations cibles telles que les client(e)s, n'ont pas été incluses dans l'étude. De ce fait, leurs perceptions et opinions n'ont pas été documentées ici. De même, la limitation à la région de Dakar ne permet pas de faire ressortir les spécificités des autres régions. Des études supplémentaires pourraient être envisagées pour élargir cette recherche. 


\section{Recommandations}

\section{État/MSAS}

- Soutenir le plaidoyer pour faire accepter les nouvelles propositions juridiques et règlementaires permettant l'offre effective de services de PF par les pharmaciens.

- Poursuivre les concertations avec tous les acteurs concernés pour explorer les voies et moyens pouvant permettre la levée rapide des obstacles relatifs à l'offre de méthodes PF par les pharmacies privées au Sénégal (MSAS, DSRSE, DPM, CNOPS, Syndicat des pharmaciens, importateurs/distributeurs, PNA, Alliance du secteur privé de la santé, partenaires techniques et financiers).

- Informer/sensibiliser les pharmaciens sur les tenants et aboutissants de cette implication, et leur expliquer le contenu du Programme PF et les attentes du ministère par rapport à ce programme.

- Prendre en compte dans les PNP des changements relatifs à l'offre de services de PF au niveau des pharmacies.

- Intégrer les pharmacies privées dans le système de santé en général (formation, approvisionnement et directives pour la prestation des services de PF, collecte des données/outils de gestion).

- Faire un plaidoyer au niveau de la Commission Économique Des Etats de l'Afrique de l'Ouest pour une révision des curricula de formation des pharmaciens afin d'y inclure un module spécifique sur la PF dans le cadre de leur formation initiale au niveau de l'université ou des écoles de formation.

- Renforcer la collaboration entre les districts sanitaires et les officines de pharmacie.

- Créer un système de supervision de l'assurance qualité.

- Initier des discussions avec tous les acteurs concernés afin d'assurer la disponibilité d'une gamme de contraceptifs dans les pharmacies, qui soit accessible de manière équitable pour les client(e)s tout en offrant des marges suffisantes pour motiver les propriétaires de pharmacies.

- Initier une étude pilote de la faisabilité de l'offre de services de PF par les pharmacies privées.

\section{Ordre et syndicat des pharmaciens}

- Soutenir le plaidoyer pour la mise en place d'un cadre juridique favorable en collaboration avec l'Ordre des pharmaciens afin de mieux impliquer le personnel des pharmacies dans l'offre initiale de services de PF.

- Contribuer au développement d'un système de supervision et de soutien technique pour les pharmaciens dans l'offre de PF.

- Encourager les pharmaciens à aménager un espace adéquat pour le counseling en PF.

- Fournir des contributions techniques à l'élaboration des outils de formation du personnel des pharmacies en PF. 


\section{Pharmaciens}

- Mettre en place un espace de confidentialité conformément à la réglementation pharmaceutique, et selon les normes de services de PF.

- Prendre les mesures nécessaires pour participer aux formations.

\section{Partenaires techniques et financiers}

- Appuyer la formation du personnel des pharmacies.

- Soutenir la mise en œuvre d'une expérience pilote relative à la faisabilité de l'offre de services de PF par les pharmacies privées.

- Fournir une assistance technique pour soutenir des actions de plaidoyer basées sur les évidences en vue d'obtenir des changements de politique en matière d'offre de services de PF au niveau des pharmacies. 


\section{Conclusion}

Les résultats de la recherche qualitative ont montré que la non-participation des pharmacies privées à l'offre de services de PF résulte d'un environnement juridique et règlementaire limitant et de contraintes non juridiques persistantes. Avec la mise en place d'un cadre juridique et réglementaire favorable, d'une bonne formation et d'un espace adéquat, le personnel des pharmacies peut faciliter l'accès des populations aux méthodes modernes de contraception.

L'implication des pharmacies privées dans l'offre de services de PF doit devenir une réalité, conformément aux engagements nationaux et internationaux du Sénégal, à l'Approche 3D et aux autres recommandations de démédicalisation, d'autant que l'Organisation Mondiale de la Santé encourage une plus grande délégation des tâches au personnel non médical, et que des expériences pilotes ont été mises en œuvre avec succès au Sénégal, en Afrique de l'Ouest et ailleurs.

Dans la dynamique de poursuivre les efforts visant à promouvoir cette pratique prometteuse à haut impact, un plaidoyer fort et urgent est nécessaire pour optimiser le rôle des pharmaciens dans l'amélioration de l'accès aux services de PF. Ce plaidoyer pourrait également aider à renforcer la visibilité, l'utilisation et l'importance de l'offre de ces services au niveau des pharmacies.

L'idée de l'offre de services de PF par les pharmacies privées est acceptable par les parties prenantes de la PF et les pharmaciens eux-mêmes, à condition que les obstacles soient levés. La prochaine étape clé serait une étude pilote pour en explorer la faisabilité.

Une synergie d'actions entre le secteur public et le secteur privé donnerait au Sénégal un plus grand potentiel pour améliorer les indicateurs dans le domaine de la SR/PF et de la survie de la mère et de l'enfant, et atteindre le plus rapidement possible les Objectifs de Développement Durable. 



\section{Références bibliographiques}

Agence Nationale de la Statistique et de la Démographie (ANSD) [Sénégal], et ICF International. 2015. Sénégal : Enquête Démographique et de Santé Continue (EDS-Continue 2014). Rockville, Maryland, USA : ANSD et ICF International.

Agence Nationale de la Statistique et de la Démographie (ANSD) [Sénégal], et ICF International. 2016. Sénégal : Enquête Démographique et de Santé Continue (EDS-Continue 2015). Rockville, Maryland, USA : ANSD et ICF International.

Décret $n^{\circ} 81-039$ du 2 février 1981 portant code de déontologie des pharmaciens.

Décret $n^{\circ}$ 96-395 du 15 mai 1996 complétant l'article 68 du décret n $81-039$ du 2 février 1981 portant code de déontologie des pharmaciens.

Direction de la Pharmacie et des Laboratoires. 2006. Politique Pharmaceutique Nationale.

Division de la Santé de la Reproduction (DSR) et Ministère de la Santé et de l'Action Sociale (MSAS). 2012. Plan d'Action National de Planification Familiale 2012-2015. République du Sénégal. Dakar : MSAS.

High-Impact Practices in Family Planning (HIP). Drug Shops and Pharmacies: Sources for family planning commodities and information. Washington, DC: USAID; 2013 Jun. Available from http://www.fphighimpactpractices.org/briefs/drug-shops-and-pharmacies

Hotchkiss et al. 2011. Effect of an expansion in private sector provision of contraceptive supplies on horizontal inequity in modern contraceptive use: evidence from Africa and Asia. International Journal for Equity in Health, 10:33.

Imaledo J. 2013. Research and recommendations on the delivery of injectable contraceptive services by Patent Medicine Vendors (PMVs) in Rural Nigeria. Policy Brief. FHI 360.

Loi n54-418 du 15 avril 1954 étendant aux territoires d'Outre-mer, au Togo et au Cameroun certaines dispositions du Code de la santé publique relatives à l'exercice de la pharmacie.

Loi 2005-18 du 5 août 2005 sur la Santé de la Reproduction.

National Population Commission (NPC) [Nigeria] and ICF International. 2014. Nigeria Demographic and Health Survey 2013. Abuja, Nigeria, and Rockville, Maryland, USA: NPC and ICF International.

Ministry of Health and Population (MOHP) [Nepal], New ERA, and ICF International Inc. 2012. Nepal Demographic and Health Survey 2011. Kathmandu, Nepal: Ministry of Health and Population, New ERA, and ICF International, Calverton, Maryland. 


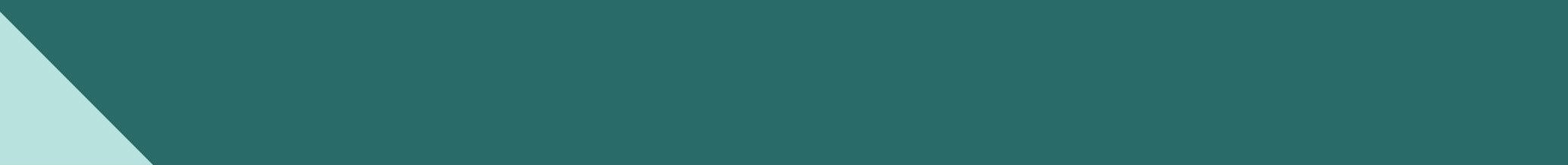

